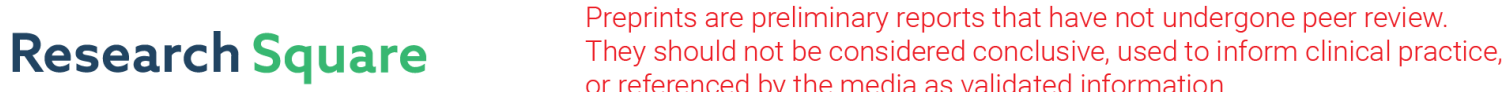 or referenced by the media as validated information. \\ The Effect of Coarctation Degrees on Wall Shear Stress Indices: An FSI Study
}

\section{Deniz Rafieianzab}

K.N.Toosi University of Technology

Mohammad Amin Abazari

K.N.Toosi University of Technology

\section{Madjid Soltani}

K.N.Toosi University of Technology

Mona Alimohammadi ( $\triangle$ mona@alimohammadi.co.uk)

K.N.Toosi University of Technology

\section{Research Article}

Keywords: Fluid-structure interaction, Aortic coarctation, WSS indices

Posted Date: January 15th, 2021

DOl: https://doi.org/10.21203/rs.3.rs-144886/v1

License: (a) This work is licensed under a Creative Commons Attribution 4.0 International License. Read Full License 


\title{
The Effect of Coarctation Degrees on Wall Shear Stress Indices: An FSI Study
}

\author{
Deniz Rafieianzab ${ }^{1}$, Mohammad Amin Abazari ${ }^{1}$, M. Soltani ${ }^{1,2,3,4,5}$, Mona Alimohammadi ${ }^{1 *}$
}

1: Department of Mechanical Engineering at K. N. Toosi Univeristy of Technology, Tehran, Iran

2: Department of Electrical and Computer Engineering, Faculty of Engineering, School of Optometry and Vision Science, Faculty of Science, University of Waterloo, Waterloo, Canada

3: Advanced Bioengineering Initiative Center, Multidisciplinary International Complex, K. N. Toosi University of Technology, Tehran, Iran

4: Centre for Biotechnology and Bioengineering (CBB), University of Waterloo, Waterloo, Ontario, Canada

5: Cancer Biology Research Center, Cancer Institute of Iran, Tehran University of Medical Sciences, Tehran, Iran

Author’s E-mails: denizrafiei77@gmail.com, m.amin.abazari@gmail.com, msoltani@uwaterloo.ca

* Corresponding author - mona@alimohammadi.co.uk

\section{Key words}

Fluid-structure interaction, Aortic coarctation, WSS indices

\begin{abstract}
Coarctation of the aorta $(\mathrm{CoA})$ is a congenital tightening of the proximal descending aorta. Flow quantification can be immensely valuable for an early and accurate diagnosis. However, there is a lack of appropriate diagnostic approaches for a variety of cardiovascular diseases, such as CoA. An accurate understanding of the disease depends on measurements of the global haemodynamics (criteria for heart function) and also the local haemodynamics (detailed data on the dynamics of blood flow). Playing a significant role in clinical processes, wall shear stress (WSS) cannot be measured clinically; thus, computation tools are needed to give an insight into this crucial haemodynamic parameter. In the present study, in order to enable the progress of non-invasive approaches that quantify global and local haemodynamics for different CoA severities, innovative computational blueprint simulations that include fluid-solid interaction (FSI) models are developed. Since there is no specific routine for managing the CoA regarding its severity, this study investigates haemodynamics in regions where clinicians do not have any information that would help physicians introduce a framework when and where initiating the intervention.
\end{abstract}




\section{Introduction}

Aortic coarctation (CoA) is a congenital heart disease (CHD). Being the fifth most common CHD [1], CoA makes up about 5-8\% of all congenital cardiac deficiencies [2,3]. It is identified by narrowing the upper descending aorta, mostly after the beginning of the left subclavian artery, affecting the blood flow from the heart to all body. As nearly $60 \%$ of the aorta's buffer capacity is located in the proximal aorta [4], this local strengthening controls the capacitance of the aorta. Moreover, stiffening and a local narrowing causes wave reflections quickly reaching the heart [5]. Afterward, it is not difficult to imagine that blood pressure distribution and perfusion changes due to the obstruction; leading to cerebral and upper body hypertension, left ventricular hypertrophy, coronary artery disease, cerebral hemorrhage, stroke, and aortic ruptures, aneurysm formation, and decreased life expectancy [6-9]. Regrettably, all available treatment strategies (i.e. surgical correction or endovascular procedures) might contribute to potential early and late complications which again influence on the future morbidity and mortality of CoA population. [10-12]. Depending on the severity of the obstruction and other comorbidities, the early course of disease might be asymptomatic, but with the $\mathrm{CoA}$ severity progression, heart failure increases among $60 \%$ of adults over 40 with untreated CoA. $75 \%$ of these patients die by 50 , and $90 \%$ die by 60 [13]. Indeed, despite advances in imaging and interventional techniques, the knowledge regarding the long-term benefits of current therapeutic approaches and the advantages of each scenario are obscure. While all of these can provide useful information on the cardiac deterioration and heart regeneration of the patients, clinical decisions are currently based on medical imaging [14], that those techniques do not address this accurately [15]. "Cardiology is flow,"[16] hence, the major causes of CoA morbidity could be described based on adverse haemodynamics. With the advance in computing power, computational simulations can provide meaningful insights into the biomechanics of CoA, presenting data behind the pressure failure observed during the Doppler Ultrasound examination, which is challenging to attain invivo. Many decades ago, O'Rourke and Cartmill proposed that the majority of unhealthiness for CoA could be described on the base of unusual haemodynamics through the ascending aorta and connected branches by indicating its alarmingly altered conduit (blood flow) and cushioning (capacity) functions linking to CoA [17]. In this research, wall shear stress (WSS) indices are applied to highlight the importance of the CoA severity. Being a vector quantity, WSS possesses both magnitude and direction-two components, affecting the advancement and progression of the aortic arch malady [18]. Effective management of CoA relies on not only the quantifications of the global haemodynamics (heart workload and 
instantaneous pressure) but also of the local haemodynamics (detailed data of the flow dynamics). Despite existing great studies on computational modeling of the coarctation of the aorta, most studies do not cover the aorta' elasticity and the fluid-structure interaction (FSI) [19-27] and/or have focused on the haemodynamic effect of CoA in patient-specific cases $[28,29]$ or on arterial WSS $[30,31]$. Studies showed that the inclusion of the vessel wall has a great impact on the blood flow parameters and WSS indices. In fact, the rigid wall design overestimates the time-averaged wall shear stress (TAWSS) by more than $50 \%$ [32]. The complexity of aortic flow patterns, choosing relevant and realistic boundary conditions, imitating vascular compliance, and the vessel's motion all contribute to the rarity of such studies. The present study evaluates the proportion of flow post-coarctation to better understand the affected and altered haemodynamic parameters caused by the area reduction in the throat of CoA that would help clinicians introduce the intervention framework, which has not been investigated before. 


\section{Methods}

Aortic geometry:

Three coarctation degrees of mild, moderate, and severe have been considered, and their haemodynamic parameters have been compared. The main concept of the geometry is taken from the study (Larissa Hütter et al.) [33].The relevant tapering of each geometry has been applied based on the cross-sectional area with $25 \%, 50 \%$, and $75 \%$ narrowing demonstrated in fig. 1 . The degree of coarctation (CD) was defined as a ratio of cross-sectional areas: $\mathrm{CD}=100 \% \times(1-$ $\left.A_{C O A} / A_{\text {Desc }}\right)$, where $A_{C O A}$ and $A_{\text {Desc }}$ are the cross-sectional areas at the coarctation and at the descending aorta, respectively. Efforts have been made to ensure that three models are the same except in their severity of narrowing.
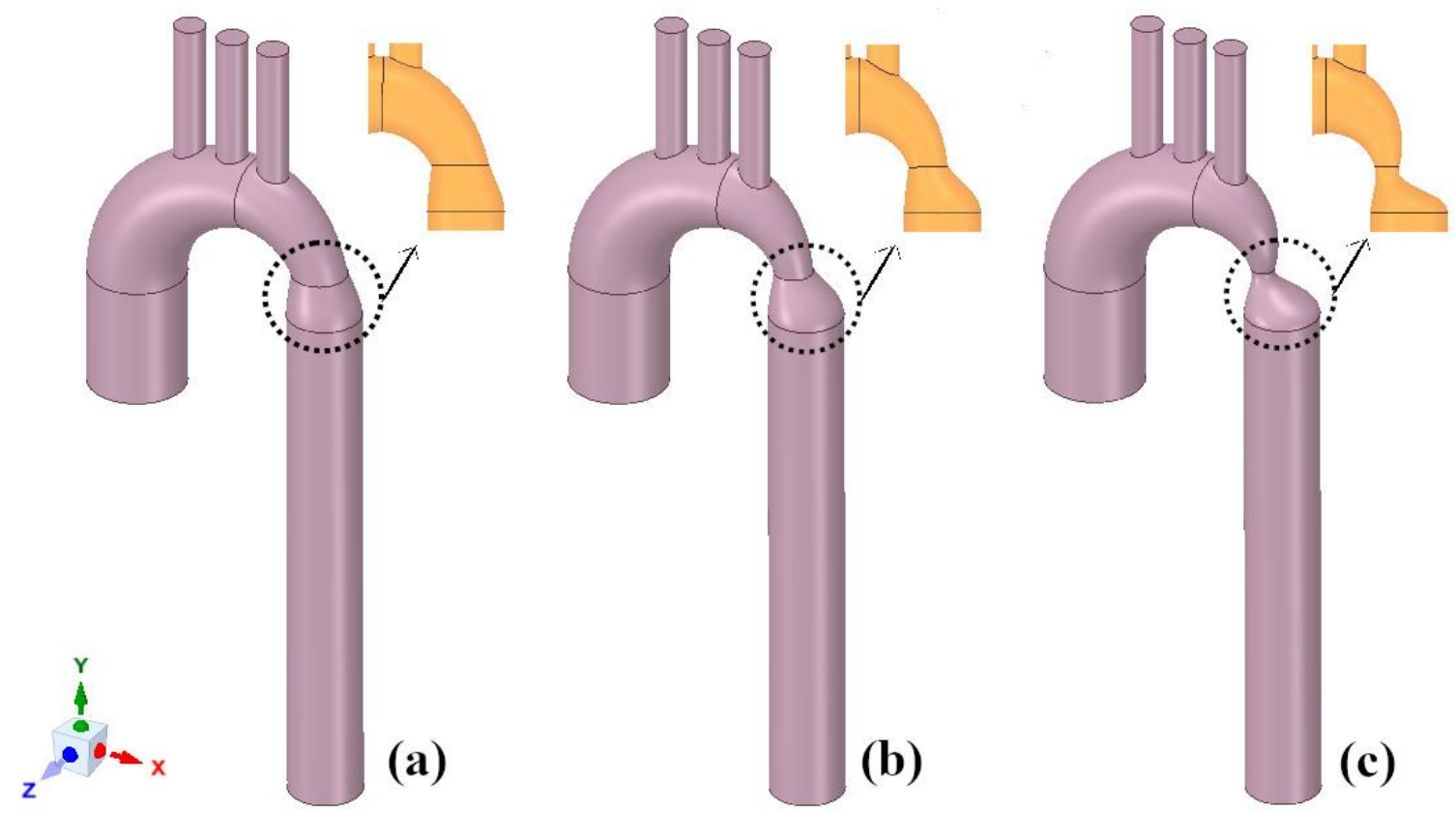

Figure 1. Three idealised geometries. (a) 25\% coarctation; (b) 50\% coarctation; and (c) $75 \%$ coarctation 
Boundary conditions:

The governing equation for the blood flow and the motion of the vessel wall is solved using ANSYS-CFX (ANSYS Inc., PA, USA), providing two-way FSI models for all three cases. For the fluid domain, about 65,000 high-quality tetrahedral mesh elements with five prism layers, and a total thickness of $1 \mathrm{~mm}$ and 1.2 growth rate have been generated using Ansys meshing. For the sensitivity analysis, two more mesh elements were calculated; however, the current model was chosen due to its accuracy and computational efficacy. For the purpose of additional accuracy and saving computational time, a mesh refinement block is deployed on the coarctation region, providing an additional refinement in the flow domain distal to the CoA. As Doormaal et al. indicated the superiority of using magnetic resonance imaging (MRI) based inlet velocity profiles over an idealised profile in the mouse aortic arch to obtain more accurate results of haemodynamic distributions [34], the same measured time-varying flow rate profiles and pressure waves were mapped as boundary conditions to the ascending and descending aorta in addition to the three side branches in all three cases taken from a coarctation study [35]. The three-dimensional CFD models were discretised by tetrahedral elements to provide an accurate display of cross-section averaged measures, including flow and the loss of pressure for each branch. As resembling blood; the fluid is considered a Newtonian, incompressible fluid, with the density of $\rho=1,056 \mathrm{~kg} / \mathrm{m}^{3}$ and dynamic viscosity of $\mu=0.0035$ Pa.s [36]. For this study, as the maximum Reynolds number in all the simulations observed to be about 440, the laminar flow was chosen. And the time step size is $0.04 \mathrm{~s}$. Mass Flow Rate and Pressure waves were modeled as a function of time (Mercuri, M.), and Vessel wall displacements were defined as a function of the blood pressures and velocities at the fluid-wall interface using FSI simulation. For all calculations, flow velocity has been assumed zero at the vessel's luminal wall, satisfying a so-called no-slip boundary condition. The continuity and NavierStokes equations solved in 3D using a transient analysis shown in "equations (1) and (2)" respectively:

$$
\begin{gathered}
\nabla \cdot \vec{u}=0 \\
\rho\left[\frac{\partial \vec{u}}{\partial t}+(u \cdot \nabla) \vec{u}+\nabla p-\mu \nabla^{2} \vec{u}\right]=0
\end{gathered}
$$

Where $\vec{u}, \rho, p$, and $\mu$ defines the vector of fluid velocity, density, the hydrostatic pressure, and viscosity of blood, respectively. Blood flow velocity and pressure, wall displacement, streamlines are shown at three cardiac-cycle points of mid- 
systole $(\mathrm{t}=0.12 \mathrm{~s})$, peak systole $(\mathrm{t}=0.2 \mathrm{~s})$, and dicrotic notch $(\mathrm{t}=0.44 \mathrm{~s})$, and after that, demonstrated and compared among the three cases. The aortic tissue's material behavior characterised using a linear elastic model obtained from the studies [37,38] (with the density of $1,160 \mathrm{~kg} / \mathrm{m}^{3}$ and young's modulus $1.08 \times$ $10^{6} \mathrm{~Pa}$ and Poisson's ratio 0.49). Wall shear stress (WSS) is the instantaneous stress applied at the wall and in order to derive a meaningful conclusion, the average of WSS (TAWSS (see for [39] definition)) over a cardiac cycle of $1.27 \mathrm{~s}$ is calculated.

$$
T A W S S=\frac{1}{T} \int|\overrightarrow{W S S(t)}| d t
$$

In order to assess the temporal oscillations in the immediate WSS vector over the cardiac cycle, OSI has been used as follows [40]:

$$
O S I=0.5\left(1-\frac{\left|\int_{0}^{T} \overrightarrow{W S S(t)} d t\right|}{\int_{0}^{T}|\overrightarrow{W S S(t)}| d t}\right)
$$

A particular index, HOLMES, is proposed in light of the signs of raised infiltration in low oscillatory areas [41] (Highly Oscillatory and Low Magnitude Shear), which is known as a useful index for linking these two features.

$$
\text { HOLMES }=\text { TAWSS }(0.5-\text { OSI })
$$

The HOLMES indicator is the modified version of TAWSS. The (0.5 - OSI) term cuts back the index in areas of low, oscillatory WSS. Besides, HOLMES offers a linear proportional index to TAWSS, which instinctively corresponds to shear features' detected effects on endothelial permeability.

The relative residence time (RRT) is calculated as [40]:

$$
\mathrm{RRT}=\frac{1}{(1-2.0 \mathrm{OSI}) . \mathrm{TAWSS}}
$$

RRT calculates the duration of residence of particles close to the wall, and it is a single measure of oscillating and low shear stress. Being inversely proportional to the magnitude of the TAWSS vector, RRT has apparent links to atherosclerosis. 


\section{Results:}

Wall Displacement:

The aortic wall's relocation for three coarctation cases is depicted in Fig.2 at three instances of time. Figure $2 \mathrm{a}$ indicates the displacement during midsystole for three coarctation degrees (left: $25 \%$ coarctation, middle: $50 \%$ coarctation, right: $75 \%$ coarctation). The ascending aorta has been displaced outwards, that its volume has increased in response to increased blood flow rate. The biggest deformation of the entry can be seen in the most severe coarctation, by up to $3.06 \mathrm{~mm}$. At peak systole, also three right anterior views of three cases are shown (Fig. 2b). A more noticeable displacement in the post-coarctation region can be observed for all cases at peak systole. The ascending aorta has expanded the most $(2.66 \mathrm{~mm})$ for all time-points except for the dicrotic notch (Figure 2c).

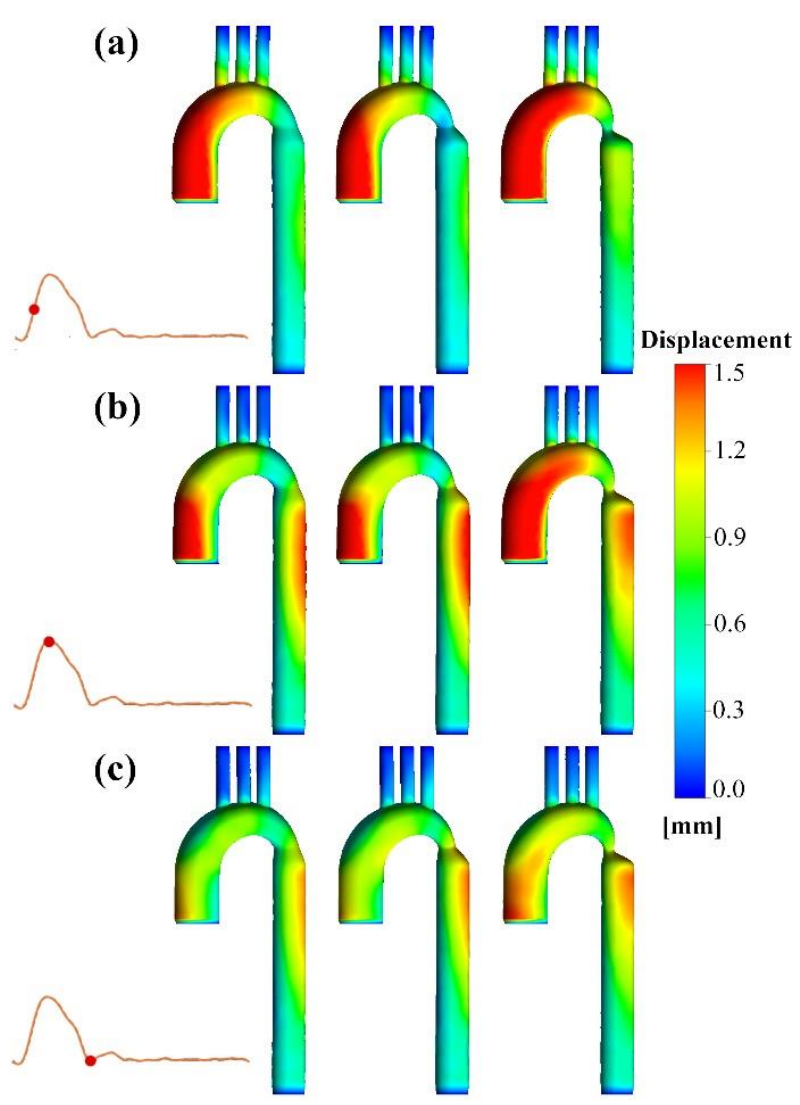

Figure 2. Vessel wall displacement during the cardiac cycle. Contours demonstrate the displacement of the geometry in the right anterior view at (a) mid-systole, (b) peak systole, and (c) dicrotic notch. (Left: $25 \%$ coarctation, middle: $50 \%$ coarctation, right: $75 \%$ coarctation)

\section{Velocity Distribution:}

Figure 3 indicates the forward and backward streamline patterns that the magnitude of the velocity of the flow is shown in color during the cardiac cycle for three different CoA degrees. During mid systole (Fig. 3a), uniform streamlines are demonstrated along the branches, aortic arch, and the CoA zone. After the coarctation, highly in the most severe case, the streamlines become less regular by a loss of symmetry, and some vortices are observed. During peak systole (Fig. 3b), uniform streamlines are seen in the aortic arch in mild case. In the moderate and severe cases, low-velocity irregular streamlines are seen after the CoA area. As the coarctation severity increases, it is clearly shown that streamlines become more irregular and more vortices have been generated, and velocity magnitude at the narrowing area increases. The separation zone right 
after the tapered part exists in all three cases. However, it's negligible for mild one and becomes more as the coarctation degree increases. During the dicrotic notch, disordered streamlines across the ascending aorta and vortices in the entry and arch and after narrowing can be seen. The considerable-scale helical flow structures have been developed in the ascending aorta (Fig. 3c). As the narrowing degree increases, irregular streamlines start affecting the branches, particularly the brachiocephalic artery, and they reach the maximum velocity magnitude of $1.1 \mathrm{~m} / \mathrm{s}$ in the severe coarctation. Figure $3 \mathrm{~d}$ shows the backward flow for three CoA cases during dicrotic notch. Stiffening and a local narrowing causes wave reflections quickly reaching the heart [5], which can be clearly seen in this study.
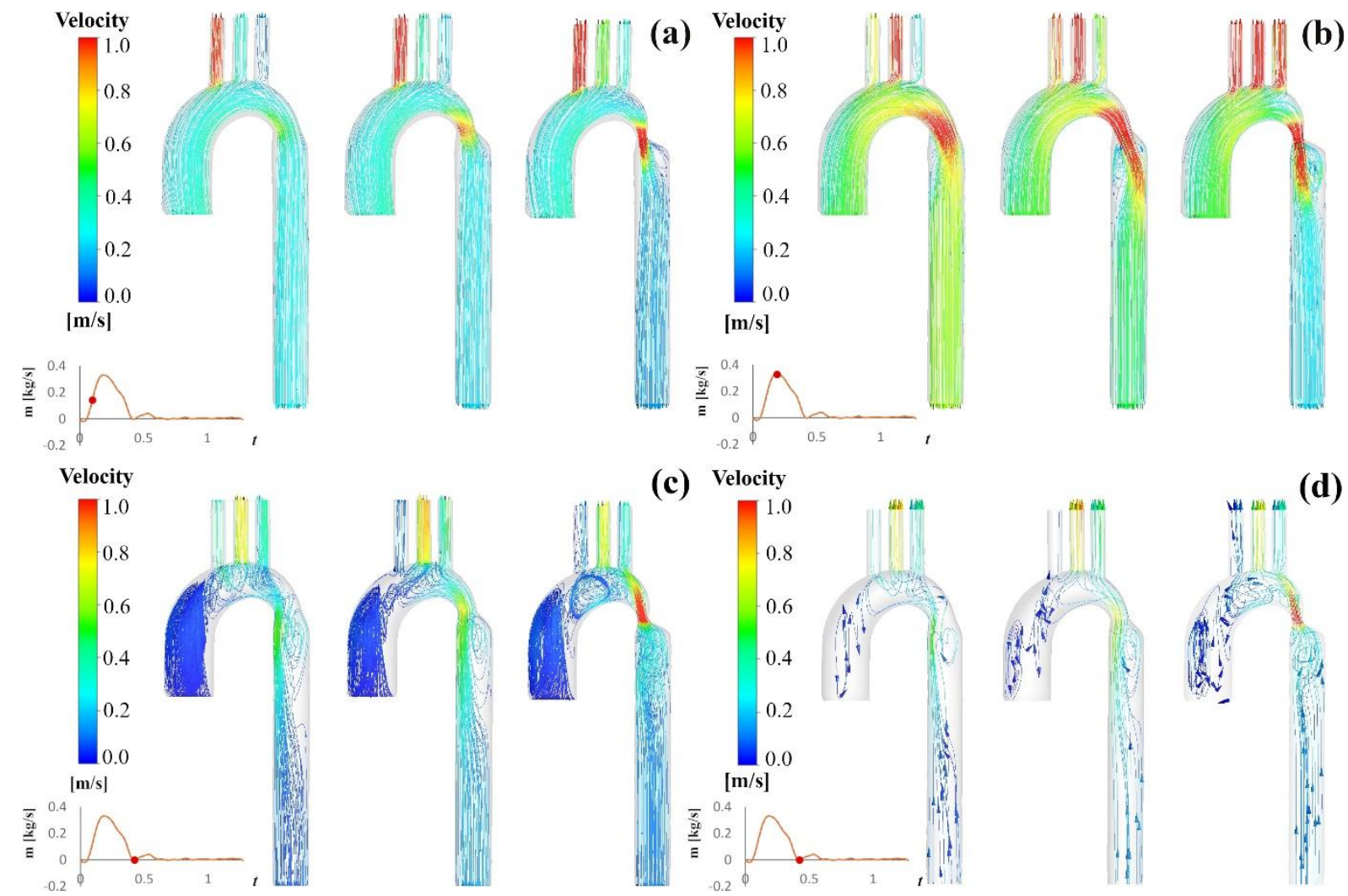

Figure 3. Streamlines in the right anterior view at (a) mid-systole, (b) peak systole, and (c) dicrotic notch; (d) Backward flow during dicrotic notch. (Left: 25\% coarctation, middle: 50\% coarctation, right: $75 \%$ coarctation) 
The velocity profile for three coarctation cases at three time instances are shown in fig.4. Blood acceleration across the coarctation region, specifically in moderate and severe cases, produced a downstream swirling, with a high-velocity jet striking the descending aorta wall. The maximal velocity increases up to $1 \mathrm{~m} / \mathrm{s}$, $1.54 \mathrm{~m} / \mathrm{s}$, and $2.45 \mathrm{~m} / \mathrm{s}$ (shown in Fig.4d), respectively for three cases, as the CoA area becomes narrower. High blood velocities are observed in the narrowing section, where the coarctation causes a reduction in the cross-sectional area. Furthermore, each supra-aortic vessel displayed evidence of increasing velocity magnitude as the coarctation became more severe.
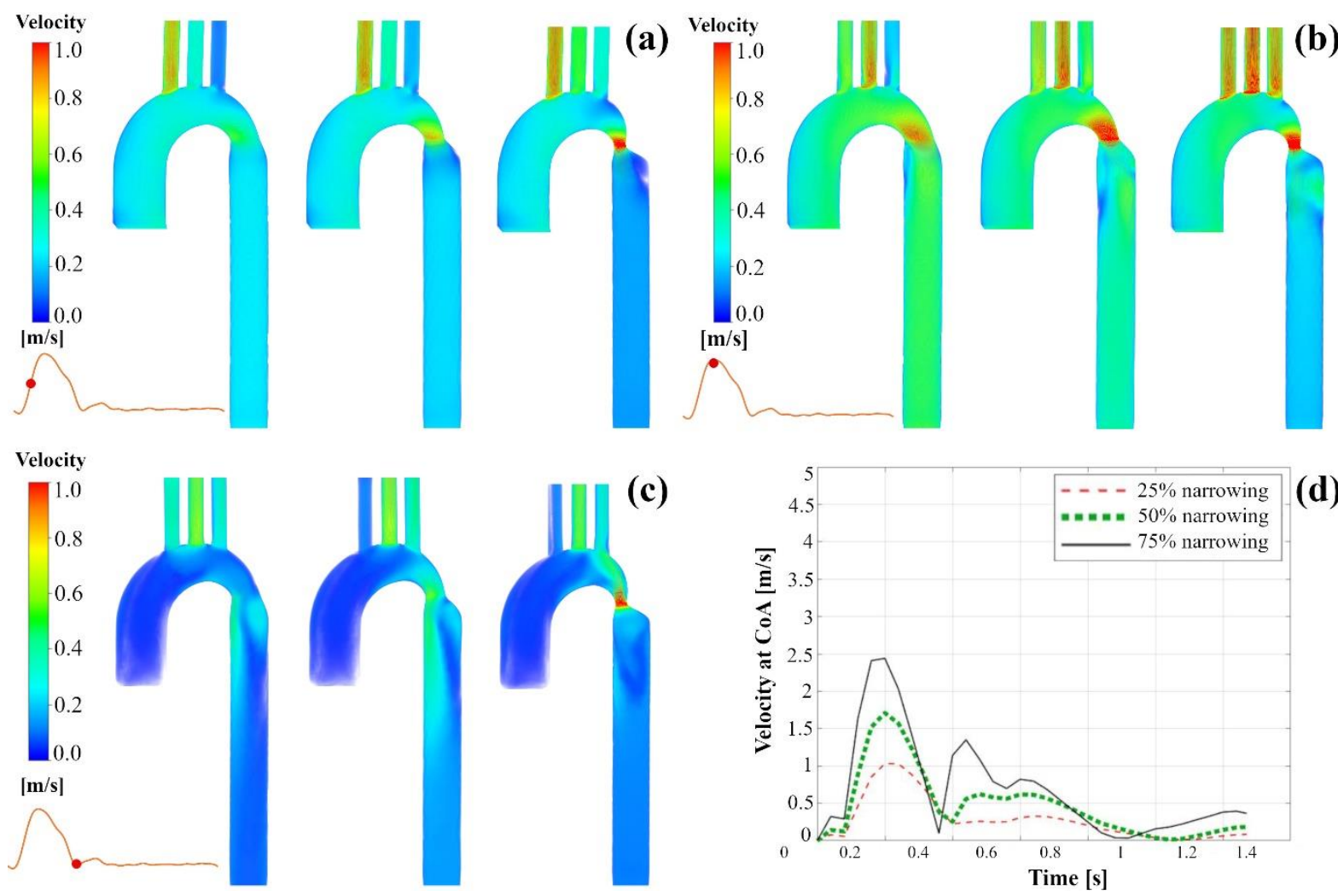

Figure 4. Velocity volume renders at various time instances. Figure shows the velocity magnitude in the right anterior view at (a) mid-systole, (b) peak systole, and (c) dicrotic notch. (Left: $25 \%$ coarctation, middle: 50\% coarctation, right: $75 \%$ coarctation; (d) Velocity at CoA in three cases against time.) 


\section{Pressure Distribution:}

The pressure distribution along the aorta is depicted during the heart cycle in fig.5. Figure 5a illustrates the distribution of pressure during mid-systole for three coarctation cases. Along with the ascending aorta, the pressure is highest, then drops at the CoA and the supra-aortic branches. As the coarctation severity increases, the difference between ascending and descending aorta's pressure becomes larger. Figure $5 \mathrm{~b}$ indicates the distribution of the pressure at the maximum systole, which is similar to that of mid-systole. However, the gradients of intraluminal pressure are more pronounced. During the dicrotic notch (Fig. 5c), the aortic arch is linked to the lower pressure, whereas the descending aorta is linked to the higher pressure. Figure $5 \mathrm{~d}$ represents the pressure at the coarctation area against time for three cases. For the mild case, the pressure is $68 \mathrm{mmHg}$ across the coarctation at peak systole. This value further reduces down to 64 and $56 \mathrm{mmHg}$ for more severe coarctation degrees, respectively. Figure 5 also shows that pressure falls sharply due to the narrowing. Along the CoA's distal end, the deceleration of flow is followed by pressure retrieval over the entire descending aorta.
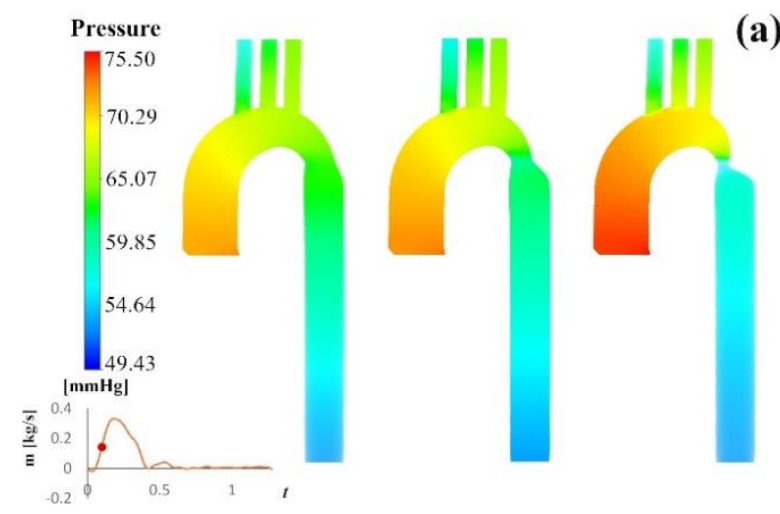

(a)
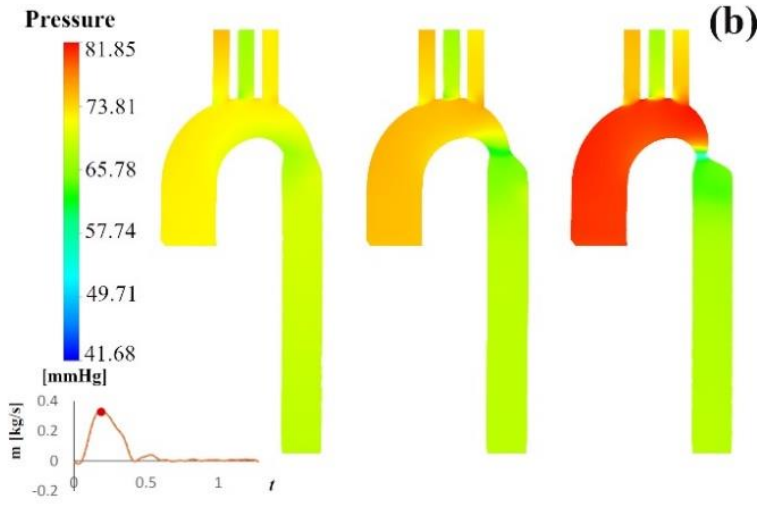

(c)

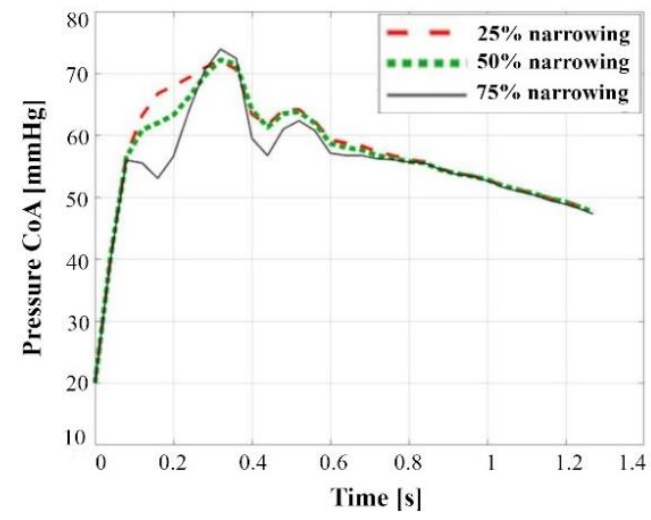

(b)

(d)
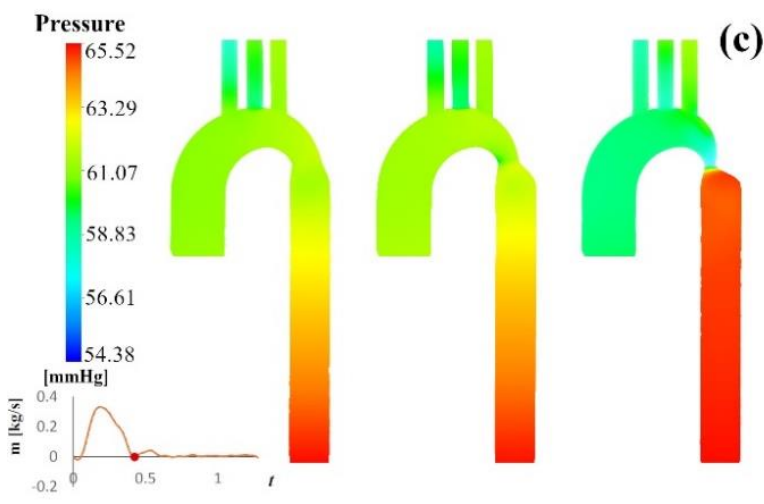

Figure 5. The distribution of pressure in the right anterior view at (a) mid systole, (b) peak systole, and (c) dicrotic notch (Left: $25 \%$ coarctation, middle: $50 \%$ coarctation, right: $75 \%$ coarctation), (d) pressure distribution in $\mathrm{CoA}$ area in three cases against time. 
Wall Shear Stress \& Oscillatory Shear Index:
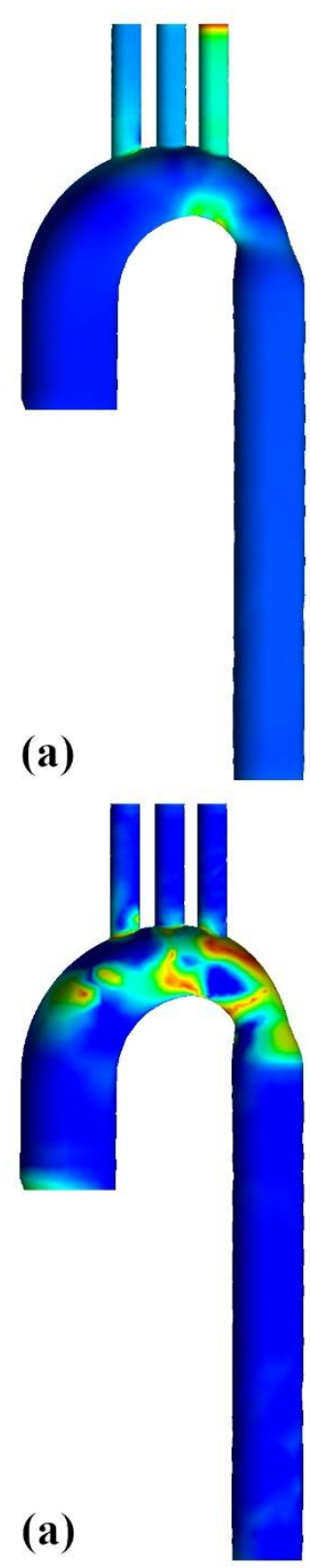
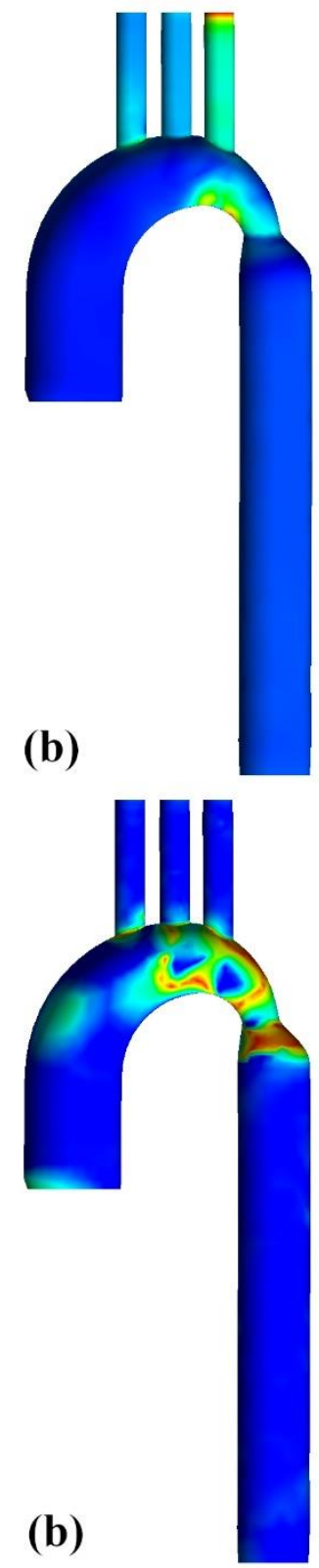

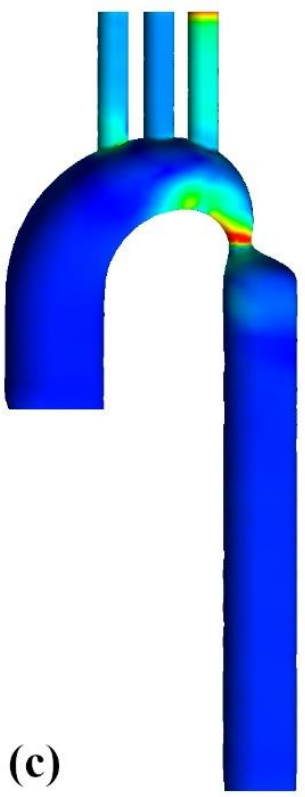

TAWSS

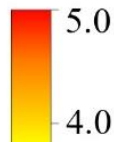

$-3.0$

$-2.0$

$-1.0$

0.0

[Pa]

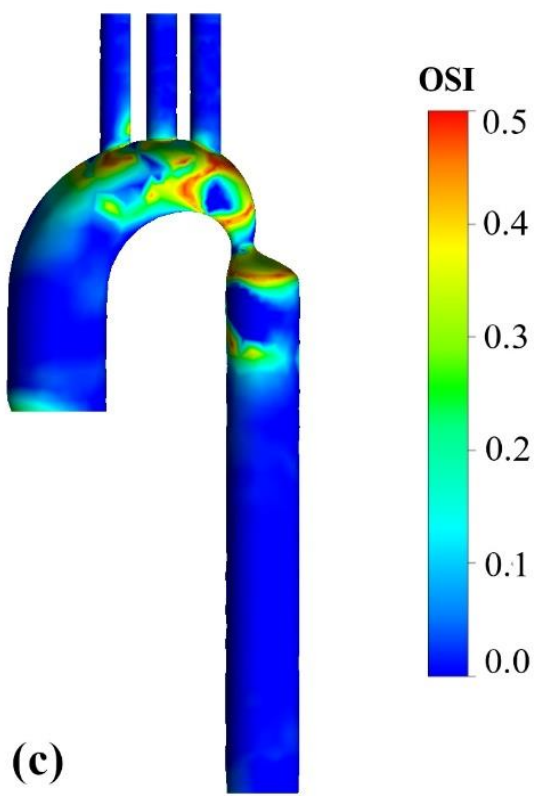

Figure 6. TAWSS and OSI characteristics for the FSI simulation. (a) 25\% coarctation (b) $50 \%$ coarctation (c) $75 \%$ coarctation.

WSS prediction is one of the key outputs from FSI simulations, usually analysed by applying indices such as TAWSS and OSI. The distribution of TAWSS, obtained with FSI simulations for three coarctation cases during the cardiac cycle, is shown in Figure 6. As much as the coarctation becomes more severe, high TAWSS magnitudes affect the narrow part more, and the difference between TAWSS magnitude before and after coarctation becomes more prominent. 
While OSI represents the WSS's changes during a cardiac cycle, it does not account for the WSS magnitude; thus, it is utilized with TAWSS. High OSI values exist at the aortic arch from the brachiocephalic artery up to the CoA zone in all three cases, which means that the changes of direction are larger than the mean flow there. In other words, areas of high OSI are generally seen in areas with low TAWSS. High OSI values also exist exactly after the narrowing part. However, the more severe the coarctation becomes, the more part after the coarctation is affected. Flow recirculation in these areas contributed to low TAWSS and high OSI. A significant reduction in OSI values after the CoA zone can be seen, particularly for moderate and severe cases.

\section{HOLMES:}

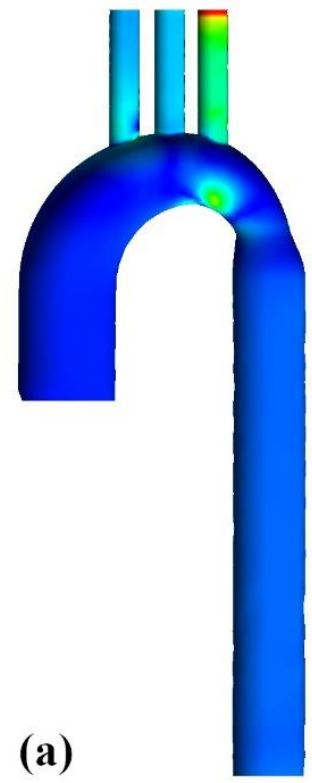

(b)

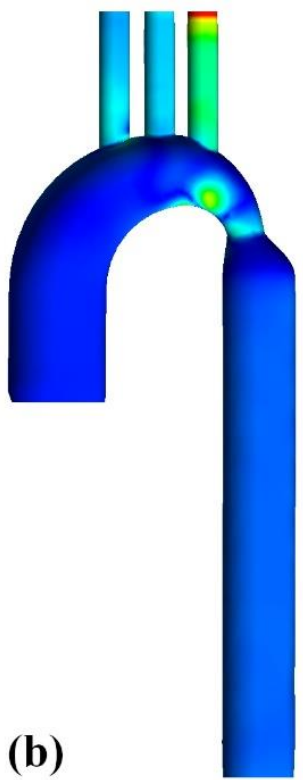

(c)
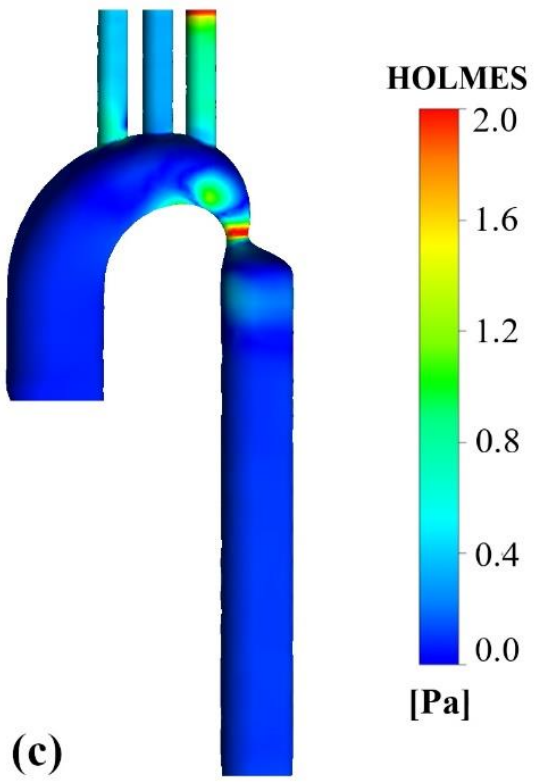

[Pa]

Figure 7. HOLMES characteristics. HOLMES distributions for the FSI simulation. (a) 25\% coarctation (b) $50 \%$ coarctation (c) $75 \%$ coarctation.

Figure 7 depicts the distribution of the HOLMES index for three cases. Areas of low HOLMES are likely to damage. The HOLMES magnitude varies before the coarctation zone, and especially, it suddenly changes in the most severe case. When comparing Fig. 5 to Fig. 6, some regions show low TAWSS and high OSI that have been recognized as high-risk areas for the rupture of an aneurysm $[43,44]$, which are shown in Fig.7 as areas with a low amount of HOLMES. 


\section{RRT:}

The contours of RRT under the threetime instances for three coarctation degrees are shown in Fig.8. RRT is increased right after the CoA and helical areas before the CoA near the left subclavian artery at mid-systole (Fig.8a), in the middle of the aortic arch at peak systole (Fig.8b), and the concave part of the arch downstream to brachiocephalic artery at the dicrotic notch while branches were also affected (figure 8c). The more severe the coarctation gets, high RRT affects the farther distances from the CoA along the descending aorta. The RRT distribution at mid-systole and peak systole were almost the same. However, some additional parts, including branches and the area near the brachiocephalic artery entry, have large RRT values at the dicrotic notch.

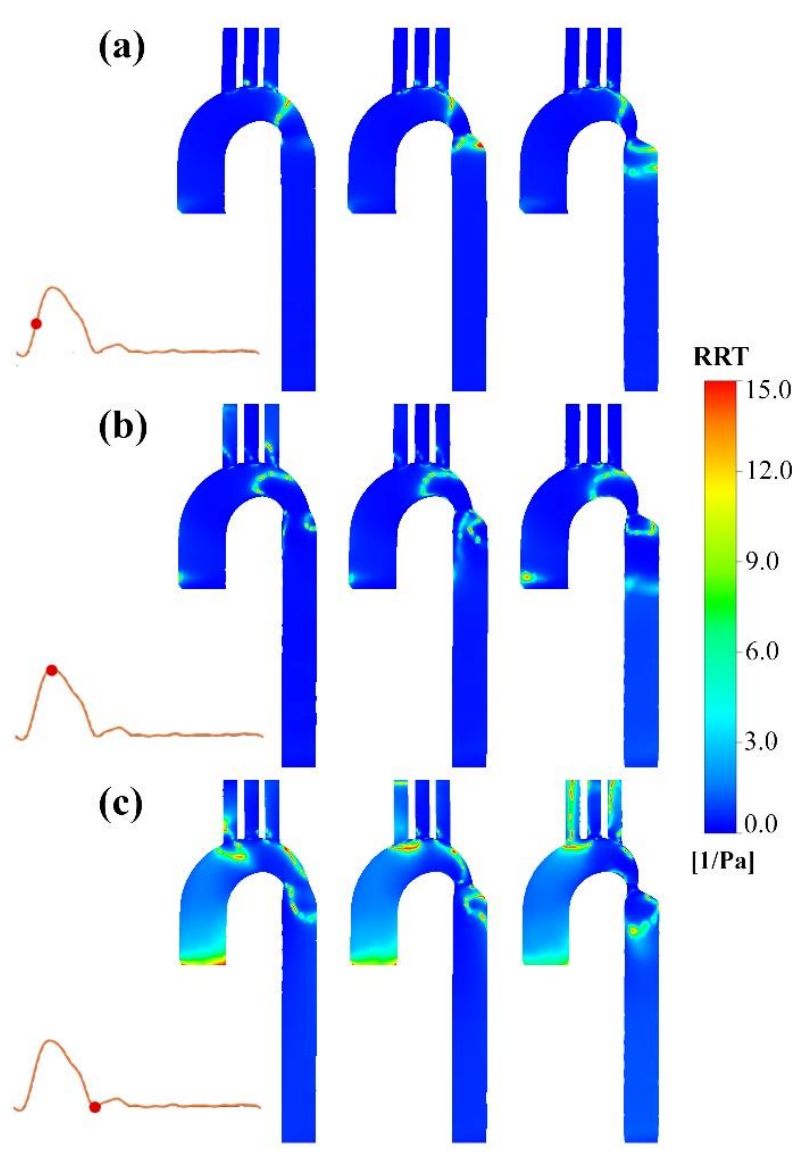

Figure 8. Contours of relative residence time (RRT) characteristics. RRT distributions for the FSI simulation (a) at mid-systole, (b) at peak systole and (c) at dicrotic notch (Left: $25 \%$ coarctation, middle: $50 \%$ coarctation, right: $75 \%$ coarctation) 


\section{Discussion}

Despite a general thought of CoA as a simple disease, evidence shows a reduced lifespan compared to the normal population [45]. Therefore, the assessment of this disease for the purpose of treatment is essential. CoA's complex flow quantification plays a critical role in precise diagnosis, assisting the physicians in optimizing the interventions and better risk stratification. To expand anatomical information, clinicians rely mainly on cardiac catheterization data to assess flow and pressure through the circulatory system, instead of sophisticated methods such as fluid dynamics, but this is invasive, high risk, and expensive [46,47]. MRI may provide a 3-D velocity field, but it is costly and impossible for many patients with a low temporal resolution [20,48-50].

In this study, three different CoA degrees have been developed, and an FSI model is applied to evaluate the CoA severity's impact on haemodynamics correctly. This is while most CoA studies are conducted under the assumption of the rigid wall, and this oversimplification can distort insights [17-25].

Arterial distensibility is an essential factor for assessing cardiovascular disorders, as raised vascular stiffness is associated with an increased risk of cardiovascular disease [6-9,51]. The smaller cross-section of the CoA zone results in higher blood velocity, contributing a more focused flow jet in the severe case that affects the aortic wall and causes dilatation of the ascending aorta [51]. The maximum displacement, located in the ascending aorta due to the heart's blood pump, is greater for the severe CoA case, which was also seen in other studies [20].

It can be seen that the narrower the CoA area, the wave reflections reach the heart more quickly. Having said that, CoA leads to a significant impedance discrepancy between the two sections of the stenotic thoracic aorta and the final aortic arch, with an increased reflection of wave [17] and quick return of the reflected pressure wave, which increases proximal pressure. Khir and Parker have reported similar findings regarding the aortic obstruction in dogs [52].

As the coarctation severity progresses, the pressure difference between ascending and descending aorta increases. Earlier results of a study in dogs with synthetic $\mathrm{CoA}$ also confirmed that the aortic pressure during peak systolic increased considerably with the aorta's progressive coarctation [53].

An intense decrease in flow through the thoracic aorta can also be seen, which tends to a more irregular profile. This leads to the generation downstream swirling, with a high-velocity jet striking hitting the descending aorta wall, contributing to high and unidirectional WSS vectors within the narrowing area. Findings show the indispensability of recognizing the coarctation degree due to 
the surprising TAWSS values differences in the CoA area. TAWSS increases considerably with the CoA progression, and the most apparent differences can be seen in close to zero OSI areas corresponding to high TAWSS values, located precisely after the coarctation area. Also, others have reported the OSI's inability to capture disturbances in areas of elevated TAWSS, which eventually cause rupture $[43,44,54]$.

As low TAWSS and raised OSI are associated with those likely to atherosclerotic plaque development [55-57], it can be realized that two particular areas are in danger of plaques creation: before the coarctation area with a specific distance, which does not change with the change of the CoA degree, and immediately after the narrowing, which expands as the coarctation becomes more severe. In regard to the first area, The helical regions in which TAWSS magnitude suddenly decreases and OSI increases are at the aortic arch near the left subclavian artery, associated with high RRT, which is seen in other studies too [54,58-60]. This favors cell-cell interactions and strengthens cell adhesion probability on vessel walls [61]. Regarding the latter area, the flow separates from the aortic wall exactly after leaving the tapered region. It goes through vortex formation leading to low-velocity flow, which eventually provoked low and multidirectional WSS vectors, leading to high RRT at the descending aorta, seen in other studies too [62-64].

A combined index named HOLMES, besides the regular TAWSS or OSI, is applied to indicate highly oscillatory, low WSS areas, in agreement with many findings suggesting these locations as especially harmful for the endothelium [55]. The degree of narrowing caused HOLMES reduction in the descending aorta significantly (up to $75 \%$ ).

The coarctation degree must be recognized thoroughly; the accuracy of evaluating the pressure drop causing by the aortic coarctation is linked to the discrimination against the narrowing intensity. Additionally, different coarctation degrees require different long-term treatment methods as considerable dissimilarities between various constrictions' severities have been shown in this study. 


\section{Limitations:}

The patient-specific assessment could not be done due to the different degrees of stenosis that cannot be applied to each patient.

\section{Data availability}

\section{All data used for this study are available from the author upon request. References:}

Suradi, H. \& Hijazi, Z. M. Current management of coarctation of the aorta. Global Cardiology Science and Practice 2015, 44 (2015).

Reller, M. D., Strickland, M. J., Riehle-Colarusso, T., Mahle, W. T. \& Correa, A. Prevalence of congenital heart defects in metropolitan Atlanta, 1998-2005. The Journal of pediatrics 153, 807-813 (2008).

Hoffman, J. I. \& Kaplan, S. The incidence of congenital heart disease. Journal of the American college of cardiology 39, 1890-1900 (2002).

Stergiopulos, N., Segers, P. \& Westerhof, N. Use of pulse pressure method for estimating total arterial compliance in vivo. American Journal of Physiology-Heart and Circulatory Physiology 276, H424-H428 (1999).

Van den Wijngaard, J. P., Siebes, M. \& Westerhof, B. E. Comparison of arterial waves derived by classical wave separation and wave intensity analysis in a model of aortic coarctation. Medical \& biological engineering \& computing 47, 211-220 (2009).

Agrawal, H., Bokowski, J. W. \& Kenny, D. Coarctation of the Aorta. Visual Guide to Neonatal Cardiology, 260 (2018).

Brown, M. L. et al. Coarctation of the aorta: lifelong surveillance is mandatory following surgical repair. Journal of the American College of Cardiology 62, 1020-1025 (2013).

Cardiology, E. b. t. A. f. E. P. et al. ESC Guidelines for the management of grown-up congenital heart disease (new version 2010) The Task Force on the Management of Grown-up Congenital Heart Disease of the European Society of Cardiology (ESC). European heart journal 31, 2915-2957 (2010).

Bouchart, F. et al. Coarctation of the aorta in adults: surgical results and long-term follow-up. The Annals of thoracic surgery 70, 1483-1488 (2000).

Lemaire, A. et al. Late complication after repair of aortic coarctation. Asian Cardiovascular and Thoracic Annals 23, 423-429 (2015).

Roos-Hesselink, J. et al. Aortic valve and aortic arch pathology after coarctation repair. Heart 89, 10741077 (2003).

Cohen, M., Fuster, V., Steele, P., Driscoll, D. \& McGoon, D. Coarctation of the aorta. Long-term followup and prediction of outcome after surgical correction. Circulation 80, 840-845 (1989).

Brickner, M. E., Hillis, L. D. \& Lange, R. A. Congenital heart disease in adults. New England Journal of Medicine 342, 334-342 (2000).

Di Carli, M. F., Geva, T. \& Davidoff, R. The future of cardiovascular imaging. Circulation 133, 2640-2661 (2016).

de Lezo, J. S. et al. Immediate and follow-up findings after stent treatment for severe coarctation of aorta. The American journal of cardiology 83, 400-406 (1999).

Richter, Y. \& Edelman, E. R. (Am Heart Assoc, 2006).

O'ROURKE, M. F. \& CARTMILL, T. B. Influence of aortic coarctation on pulsatile hemodynamics in the proximal aorta. Circulation 44, 281-292 (1971).

Cecchi, E. et al. Role of hemodynamic shear stress in cardiovascular disease. Atherosclerosis 214, 249256 (2011).

Keshavarz-Motamed, Z. \& Kadem, L. 3D pulsatile flow in a curved tube with coexisting model of aortic stenosis and coarctation of the aorta. Medical engineering \& physics 33, 315-324 (2011).

LaDisa, J. F. et al. Computational simulations for aortic coarctation: representative results from a sampling of patients. Journal of biomechanical engineering 133 (2011).

Olivieri, L. J. et al. Hemodynamic modeling of surgically repaired coarctation of the aorta. Cardiovascular engineering and technology 2, 288-295 (2011). 
Valverde, I. et al. Predicting hemodynamics in native and residual coarctation: preliminary results of a rigid-wall computational-fluid-dynamics model (RW-CFD) validated against clinically invasive pressure measures at rest and during pharmacological stress. Journal of Cardiovascular Magnetic Resonance 13, 1-4 (2011). initial findings and application to aortic coarctation. Medical engineering \& physics 35, 723-735 (2013). Goubergrits, L. et al. The impact of MRI-based inflow for the hemodynamic evaluation of aortic coarctation. Annals of biomedical engineering 41, 2575-2587 (2013). Zakerzadeh, R., Hsu, M.-C. \& Sacks, M. S. Computational methods for the aortic heart valve and its replacements. Expert review of medical devices 14, 849-866 (2017). Mercuri, M. et al. Subject-specific simulation for non-invasive assessment of aortic coarctation: Towards a translational approach. Medical engineering \& physics 77, 69-79 (2020). $\mathrm{Ha}, \mathrm{H}$. et al. Estimating the irreversible pressure drop across a stenosis by quantifying turbulence production using 4D Flow MRI. Scientific reports 7, 46618 (2017). $\mathrm{Kim}, \mathrm{H}$. J. et al. On coupling a lumped parameter heart model and a three-dimensional finite element aorta model. Annals of biomedical engineering 37, 2153-2169 (2009). Tombetti, E. et al. Novel angiographic scores for evaluation of large vessel vasculitis. Scientific reports 8, 1-11 (2018).

30 Park, H., Park, J. H. \& Lee, S. J. In vivo measurement of hemodynamic information in stenosed rat blood vessels using X-ray PIV. Scientific reports 6, 37985 (2016). Coogan, J. S., Humphrey, J. D. \& Figueroa, C. A. Computational simulations of hemodynamic changes within thoracic, coronary, and cerebral arteries following early wall remodeling in response to distal aortic coarctation. Biomechanics and modeling in mechanobiology 12, 79-93 (2013). Alimohammadi, M. et al. Aortic dissection simulation models for clinical support: fluid-structure interaction vs. rigid wall models. Biomedical engineering online 14, 1-16 (2015). Hütter, L. et al. Fabrication of a compliant phantom of the human aortic arch for use in Particle Image Velocimetry (PIV) experimentation. Current Directions in Biomedical Engineering 2, 493-497 (2016). Van Doormaal, M. A. et al. Haemodynamics in the mouse aortic arch computed from MRI-derived velocities at the aortic root. Journal of The Royal Society Interface 9, 2834-2844 (2012). Mercuri, M. Tuning of boundary conditions parameters for hemodynamics simulation using patient data, University of Sheffield, (2019).

(Springer, 2018).

37 Vlachopoulos, C., O'Rourke, M. \& Nichols, W. W. McDonald's blood flow in arteries: theoretical, experimental and clinical principles. (CRC press, 2011).

38 Akai, T. et al. Biomechanical analysis of an aortic aneurysm model and its clinical application to thoracic aortic aneurysms for defining "saccular" aneurysms. Journal of the American Heart Association 4, e001547 (2015).

39 Taylor, C. A., Hughes, T. J. \& Zarins, C. K. Finite element modeling of three-dimensional pulsatile flow in the abdominal aorta: relevance to atherosclerosis. Annals of biomedical engineering 26, 975-987 (1998). biomedical engineering. 1-4 (IEEE).

41 Alimohammadi, M., Pichardo-Almarza, C., Agu, O. \& Díaz-Zuccarini, V. Development of a patientspecific multi-scale model to understand atherosclerosis and calcification locations: comparison with in vivo data in an aortic dissection. Frontiers in physiology 7, 238 (2016).

42 Kedem, O. \& Katchalsky, A. Thermodynamic analysis of the permeability of biological membranes to non-electrolytes. Biochimica et biophysica Acta 27, 229-246 (1958).

Sakalihasan, N., Limet, R. \& Defawe, O. D. Abdominal aortic aneurysm. The Lancet 365, 1577-1589 (2005).

44 Meng, H., Tutino, V., Xiang, J. \& Siddiqui, A. High WSS or low WSS? Complex interactions of hemodynamics with intracranial aneurysm initiation, growth, and rupture: toward a unifying hypothesis. American Journal of Neuroradiology 35, 1254-1262 (2014).

45 Menon, A. et al. A coupled experimental and computational approach to quantify deleterious hemodynamics, vascular alterations, and mechanisms of long-term morbidity in response to aortic coarctation. Journal of pharmacological and toxicological methods 65, 18-28 (2012). 

Sons, 2008).

47 Bergersen, L., Foerster, S., Marshall, A. C. \& Meadows, J. Congenital heart disease: the catheterization manual. (Springer Science \& Business Media, 2008).

48 Holloway, B. J., Rosewarne, D. \& Jones, R. G. Imaging of thoracic aortic disease. The British journal of radiology 84, S338-S354 (2011).

49 Kilner, P. J., Gatehouse, P. D. \& Firmin, D. N. Flow measurement by magnetic resonance: a unique asset worth optimising. Journal of Cardiovascular Magnetic Resonance 9, 723-728 (2007).

50 Elkins, C. J. \& Alley, M. T. Magnetic resonance velocimetry: applications of magnetic resonance imaging in the measurement of fluid motion. Experiments in Fluids 43, 823-858 (2007).

51 Itu, L. et al. Non-invasive assessment of patient-specific aortic haemodynamics from four-dimensional flow MRI data. Interface focus 8, 20170006 (2018).

52 Khir, A. \& Parker, K. Wave intensity in the ascending aorta: effects of arterial occlusion. Journal of biomechanics 38, 647-655 (2005).

GUPTA, T. C. \& Wiggers, C. J. Basic hemodynamic changes produced by aortic coarctation of different degrees. Circulation 3, 17-31 (1951).

54 Xiang, J. et al. Hemodynamic-morphologic discriminants for intracranial aneurysm rupture. Stroke 42, 144-152 (2011).

55 Wentzel, J. J. et al. Does shear stress modulate both plaque progression and regression in the thoracic aorta?: Human study using serial magnetic resonance imaging. Journal of the American College of Cardiology 45, 846-854 (2005).

Davies, P. F. Hemodynamic shear stress and the endothelium in cardiovascular pathophysiology. Nature clinical practice Cardiovascular medicine 6, 16-26 (2009).

57 Frydrychowicz, A. et al. Three-dimensional analysis of segmental wall shear stress in the aorta by flowsensitive four-dimensional-MRI. Journal of Magnetic Resonance Imaging: An Official Journal of the International Society for Magnetic Resonance in Medicine 30, 77-84 (2009).

58 Menichini, C., Cheng, Z., Gibbs, R. G. \& Xu, X. Y. Predicting false lumen thrombosis in patient-specific models of aortic dissection. Journal of The Royal Society Interface 13, 20160759 (2016).

59 Sun, A. et al. Does lower limb exercise worsen renal artery hemodynamics in patients with abdominal aortic aneurysm? PloS one 10, e0125121 (2015).

60 Hoi, Y., Zhou, Y.-Q., Zhang, X., Henkelman, R. M. \& Steinman, D. A. Correlation between local hemodynamics and lesion distribution in a novel aortic regurgitation murine model of atherosclerosis. Annals of biomedical engineering 39, 1414-1422 (2011).

61 Ku, D. N., Giddens, D. P., Zarins, C. K. \& Glagov, S. Pulsatile flow and atherosclerosis in the human carotid bifurcation. Positive correlation between plaque location and low oscillating shear stress. Arteriosclerosis: An Official Journal of the American Heart Association, Inc. 5, 293-302 (1985).

62 Guillot, M., Ascuitto, R., Ross-Ascuitto, N., Mallula, K. \& Siwik, E. Computational fluid dynamics simulations as a complementary study for transcatheter endovascular stent implantation for recoarctation of the aorta associated with minimal pressure drop: an aneurysmal ductal ampulla with aortic isthmus narrowing. Cardiology in the Young 29, 768-776 (2019).

63 Jr. LaDisa, J. F. et al. Computational simulations demonstrate altered wall shear stress in aortic coarctation patients treated by resection with end-to-end anastomosis. Congenital heart disease 6, 432-443 (2011).

64 Tan, F. et al. Fluid-structure interaction analysis of wall stress and flow patterns in a thoracic aortic aneurysm. International journal of applied mechanics 1, 179-199 (2009). 
Figure 1. Three idealised geometries. (a) 25\% coarctation; (b) 50\% coarctation; and (c) $75 \%$ coarctation

Figure 2. Vessel wall displacement during the cardiac cycle. Contours demonstrate the displacement of the geometry in the right anterior view at (a) mid-systole, (b) peak systole, and (c) dicrotic notch. (Left: $25 \%$ coarctation, middle: $50 \%$ coarctation, right: $75 \%$ coarctation)

Figure 3. Streamlines in the right anterior view at (a) mid-systole, (b) peak systole, and (c) dicrotic notch; (d) Backward flow during dicrotic notch. (Left: 25\% coarctation, middle: 50\% coarctation, right: $75 \%$ coarctation)

Figure 4. Velocity volume renders at various time instances. Figure shows the velocity magnitude in the right anterior view at (a) mid-systole, (b) peak systole, and (c) dicrotic notch. (Left: $25 \%$ coarctation, middle: $50 \%$ coarctation, right: $75 \%$ coarctation; (d) Velocity at CoA in three cases against time.)

Figure 5. The distribution of pressure in the right anterior view at (a) mid systole, (b) peak systole, and (c) dicrotic notch (Left: 25\% coarctation, middle: 50\% coarctation, right: 75\% coarctation), (d) pressure distribution in $\mathrm{CoA}$ area in three cases against time.

Figure 6. TAWSS and OSI characteristics for the FSI simulation. (a) 25\% coarctation (b) $50 \%$ coarctation (c) $75 \%$ coarctation.

Figure 7. HOLMES characteristics. HOLMES distributions for the FSI simulation. (a) 25\% coarctation (b) $50 \%$ coarctation (c) $75 \%$ coarctation.

Figure 8. Contours of relative residence time (RRT) characteristics. RRT distributions for the FSI simulation (a) at mid-systole, (b) at peak systole and (c) at dicrotic notch (Left: 25\% coarctation, middle: $50 \%$ coarctation, right: $75 \%$ coarctation)

\section{Author contributions}

M. A. and D. R. conceived the idea. D. R. carried out the simulations, analyzed the data, and drafted the manuscript. A. A. participated in data analysis. M. S. and M. A. made revisions. All authors discussed the results and approved the final manuscript.

\section{Acknowledgements}

The authors thank Dr. Parham Sadeghipour for valuable discussions and constructive comments. The study was not supported by any funding.

\section{Additional Information}

\section{Competing interests}

The authors declare no competing interests. 
Figures
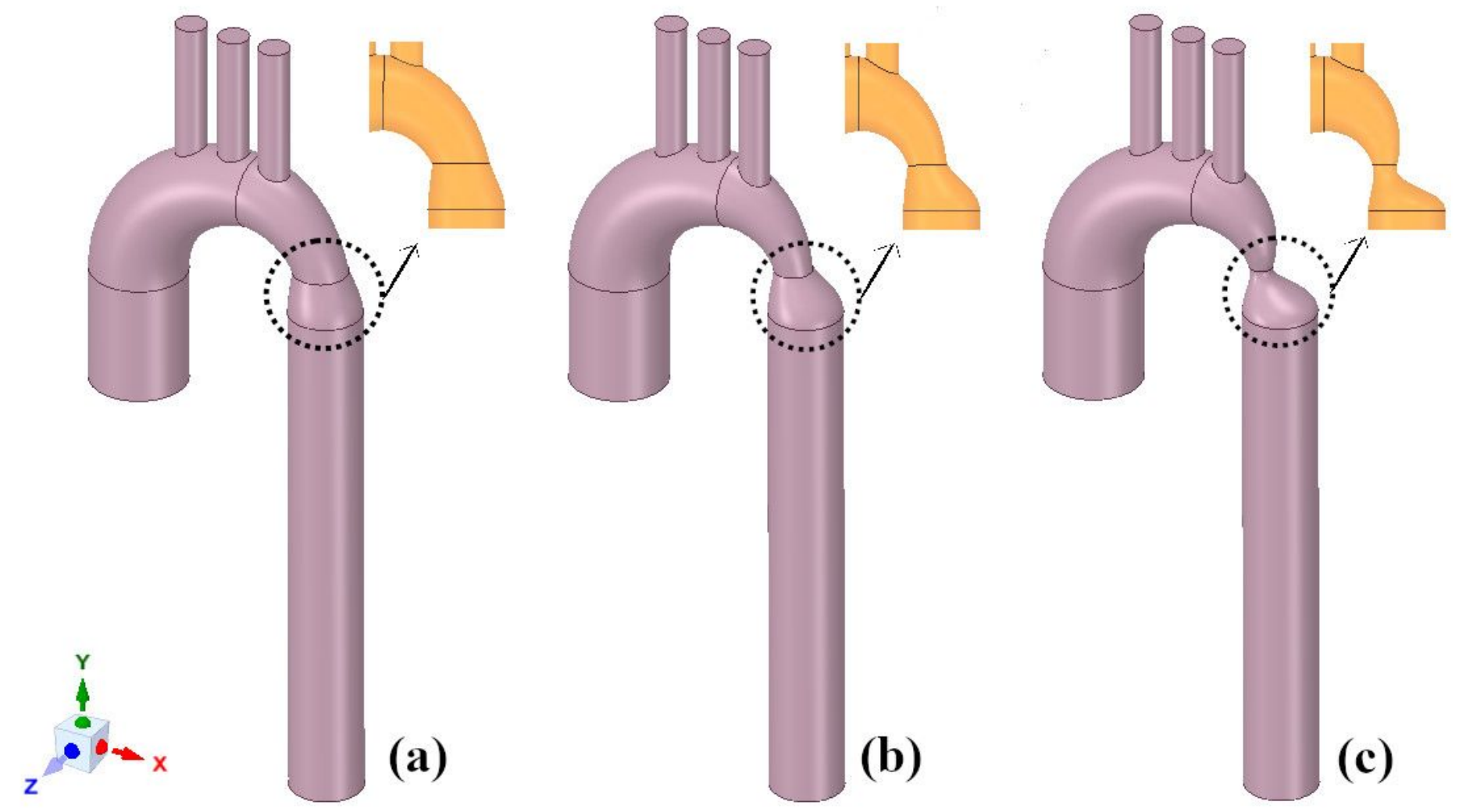

Figure 1

Three idealised geometries. (a) 25\% coarctation; (b) 50\% coarctation; and (c) $75 \%$ coarctation 


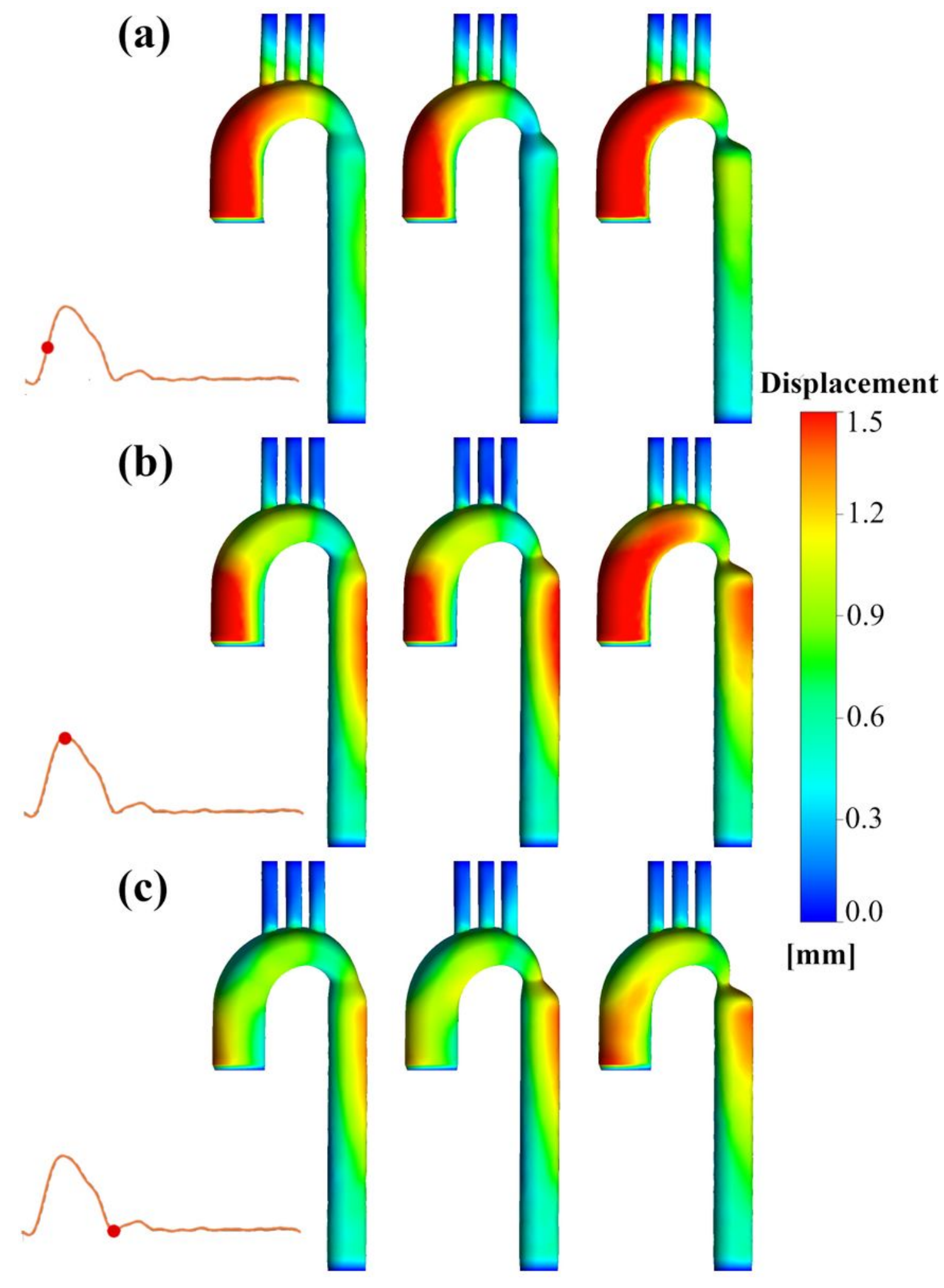

Figure 2

Vessel wall displacement during the cardiac cycle. Contours demonstrate the displacement of the geometry in the right anterior view at (a) mid-systole, (b) peak systole, and (c) dicrotic notch. (Left: $25 \%$ coarctation, middle: $50 \%$ coarctation, right: $75 \%$ coarctation) 

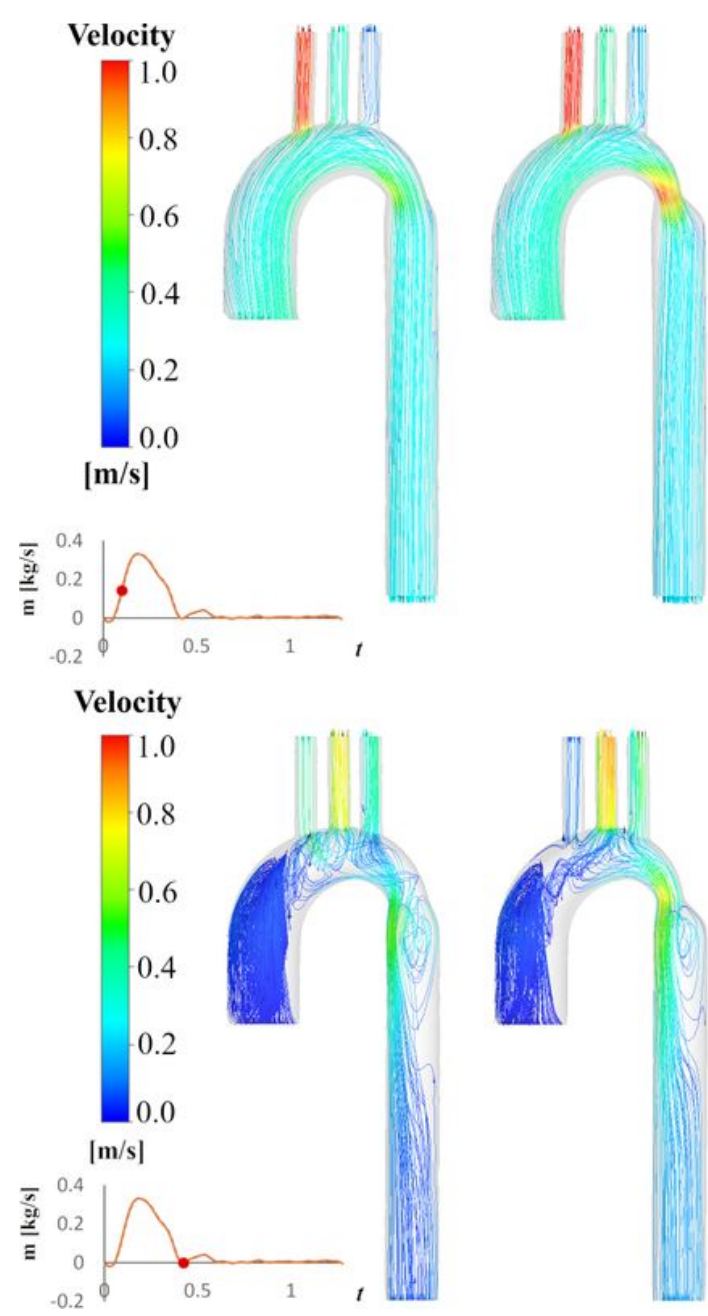

Vil (if) (a)

(a)

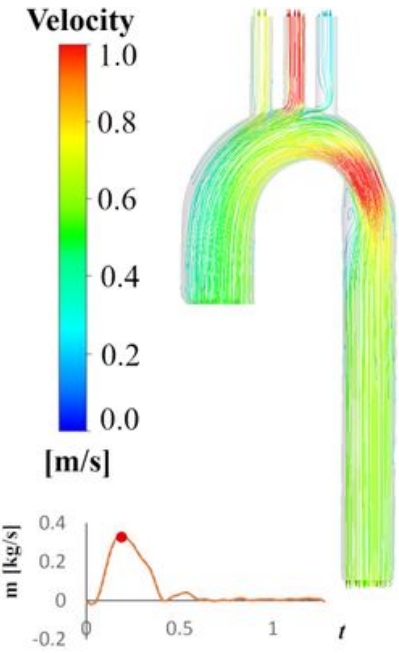

(c)
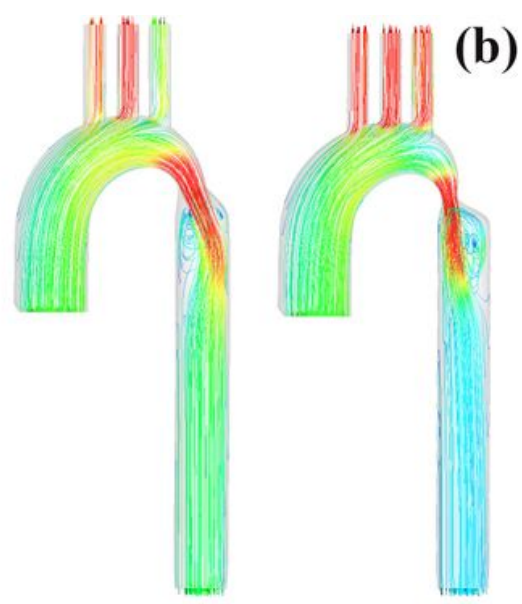

(b)

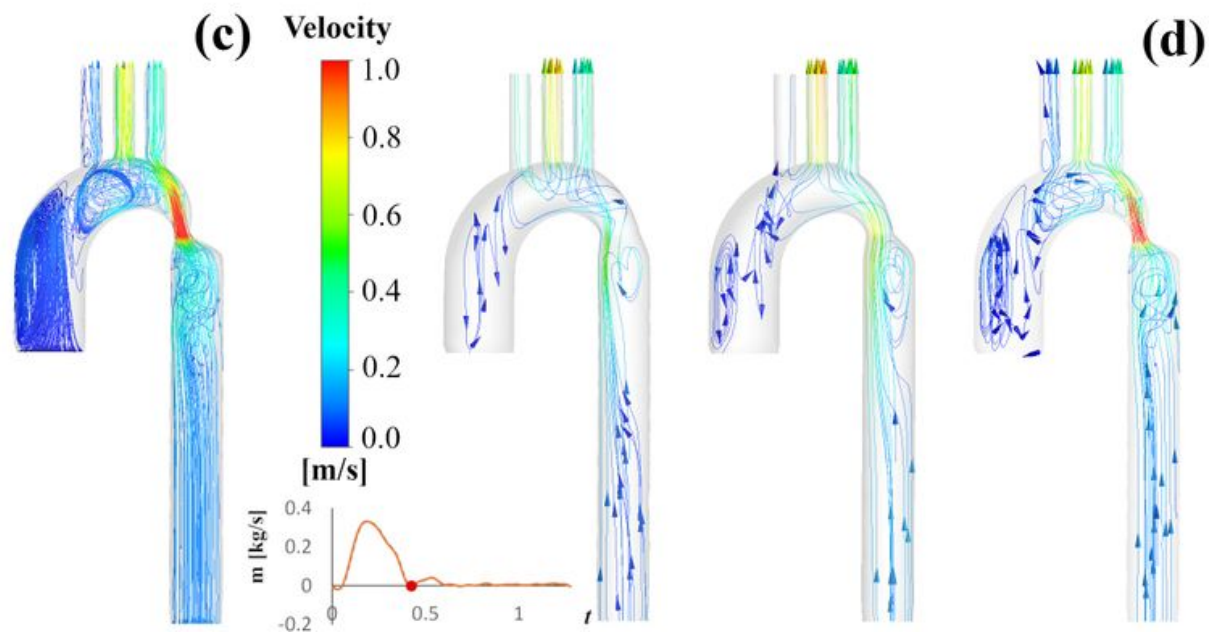

\section{Figure 3}

Streamlines in the right anterior view at (a) mid-systole, (b) peak systole, and (c) dicrotic notch; (d) Backward flow during dicrotic notch. (Left: $25 \%$ coarctation, middle: 50\% coarctation, right: $75 \%$ coarctation) 

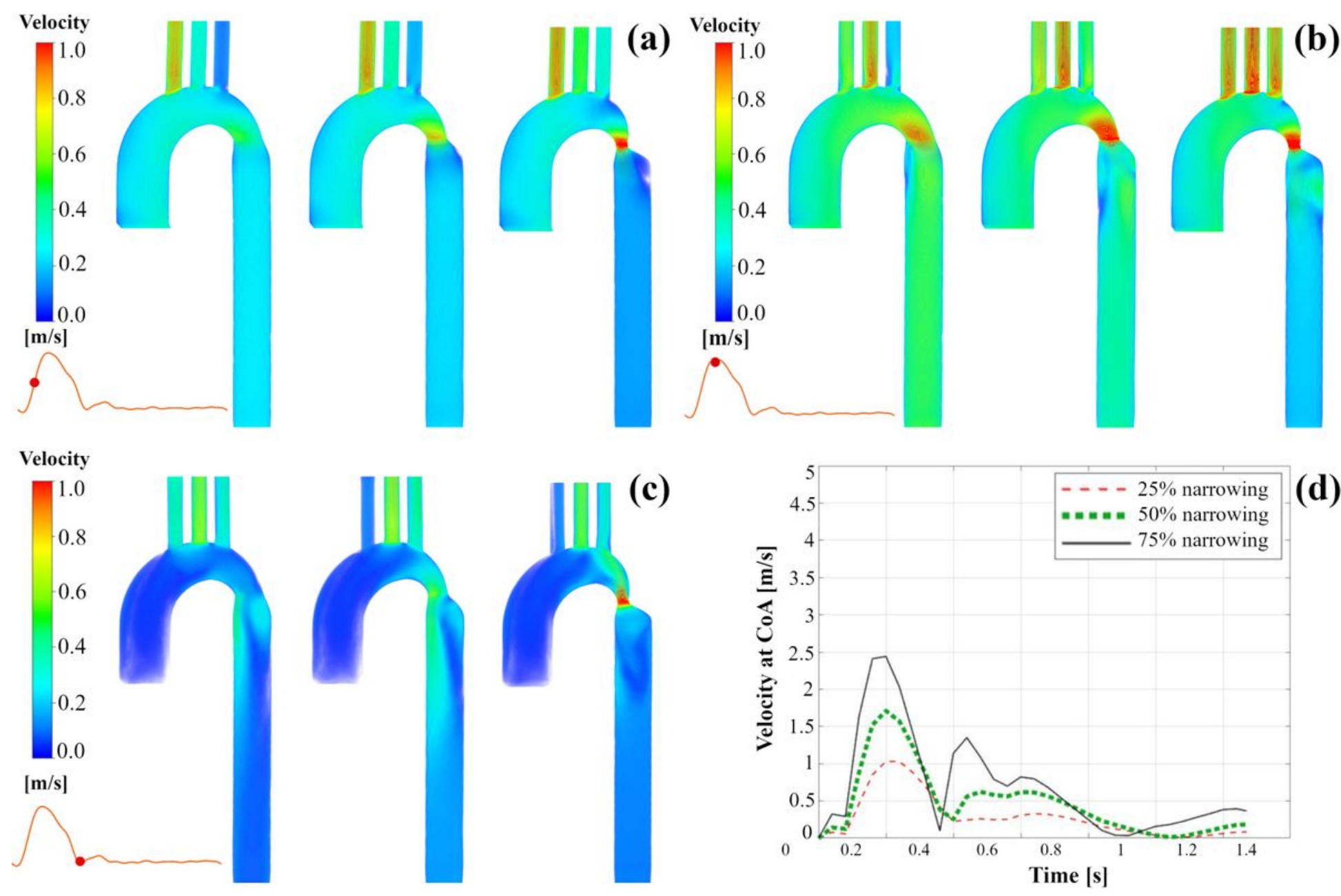

(d)

Figure 4

Velocity volume renders at various time instances. Figure shows the velocity magnitude in the right anterior view at (a) mid-systole, (b) peak systole, and (c) dicrotic notch. (Left: $25 \%$ coarctation, middle: $50 \%$ coarctation, right: $75 \%$ coarctation; (d) Velocity at CoA in three cases against time.) 

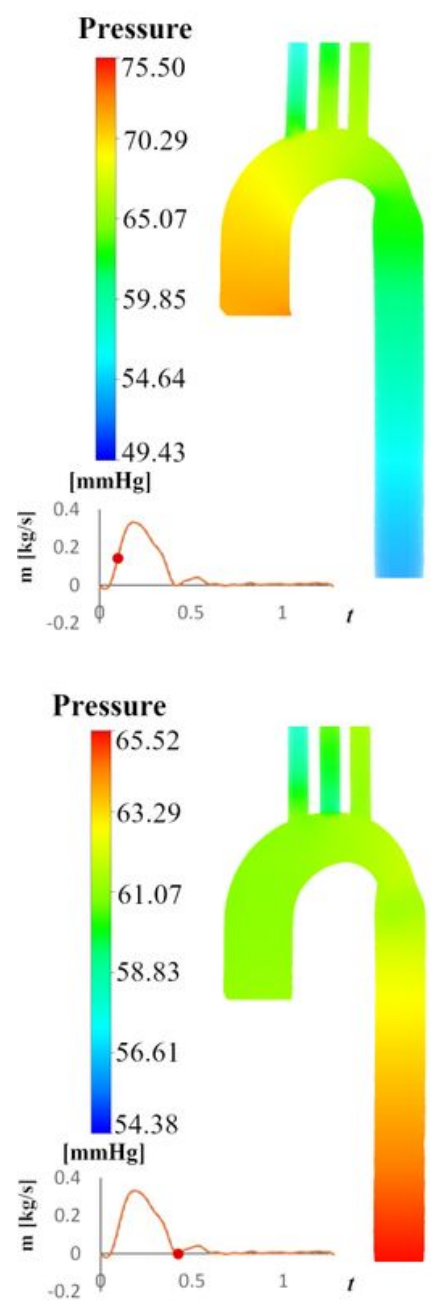

(a)
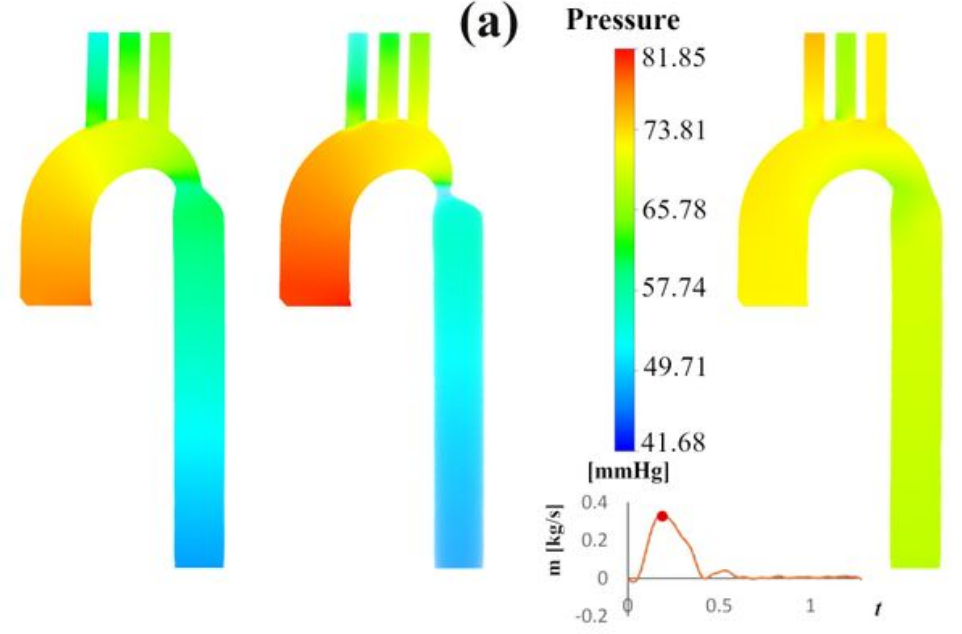
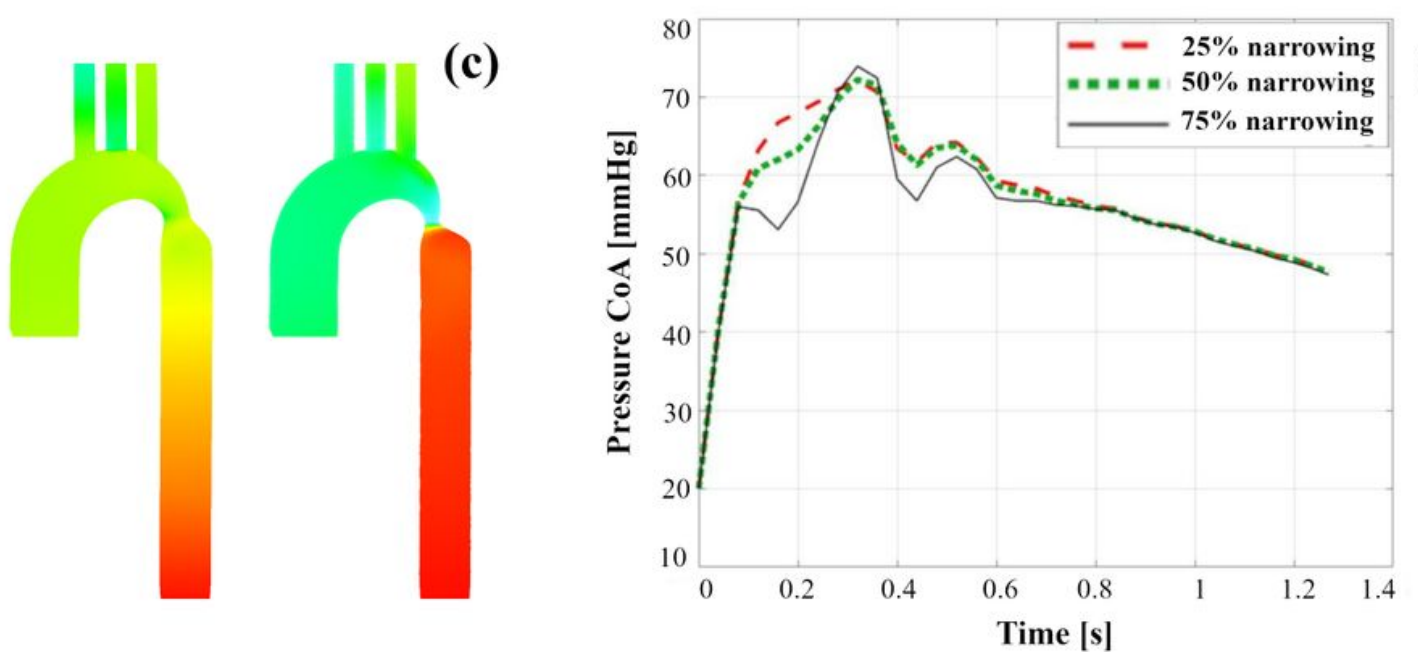

(d)

Figure 5

The distribution of pressure in the right anterior view at (a) mid systole, (b) peak systole, and (c) dicrotic notch (Left: $25 \%$ coarctation, middle: $50 \%$ coarctation, right: $75 \%$ coarctation), (d) pressure distribution in CoA area in three cases against time. 

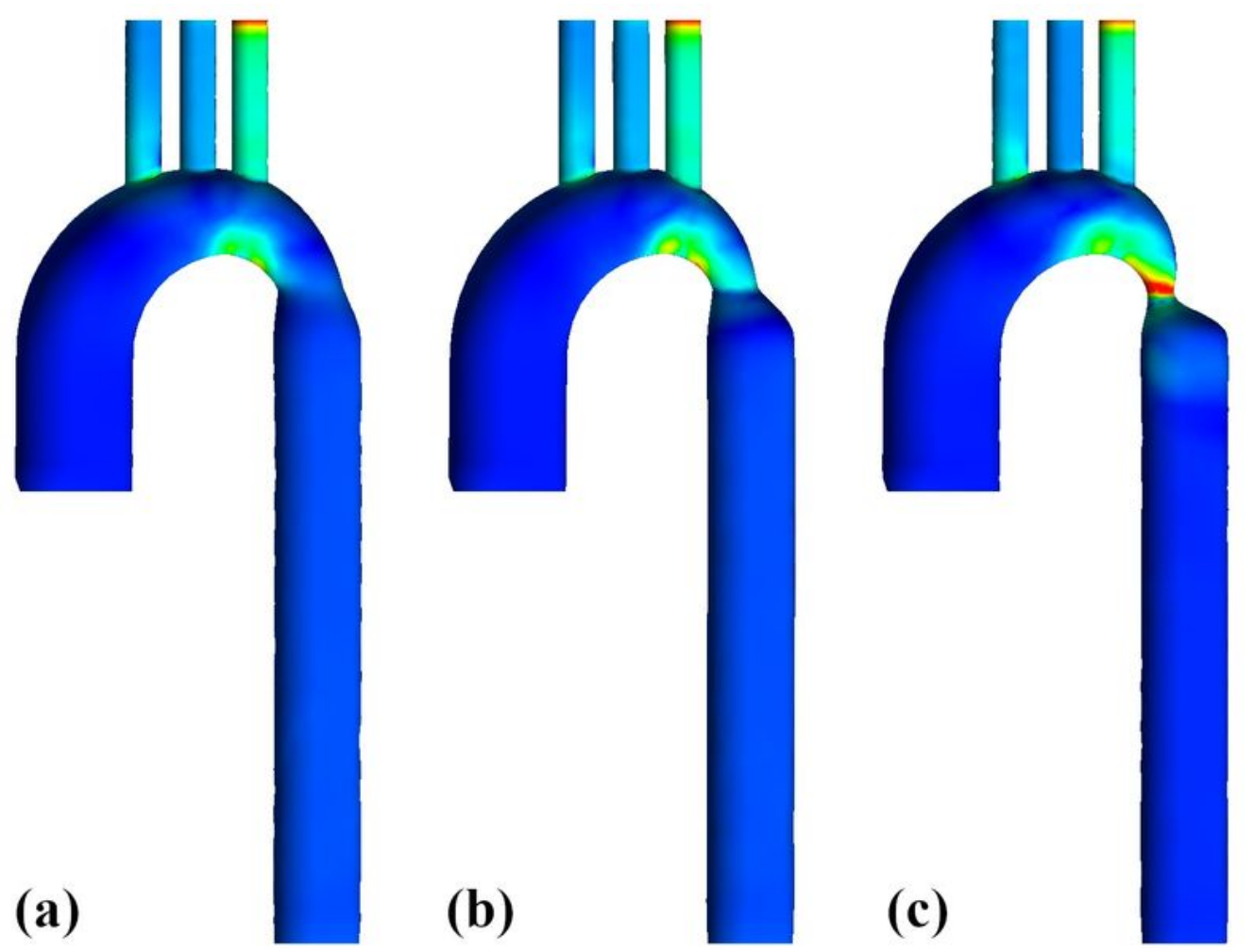

\section{TAWSS}
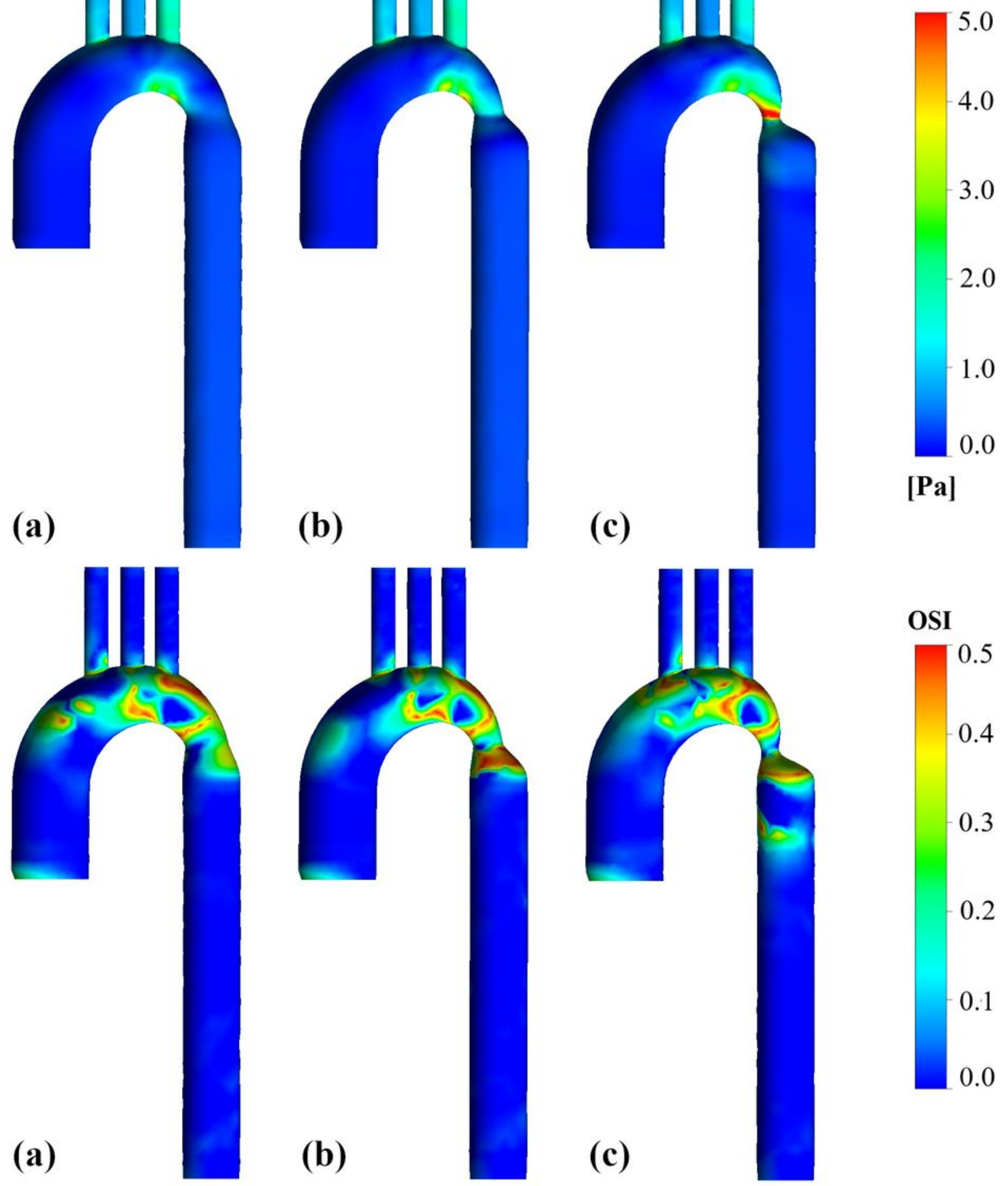

\section{OSI}

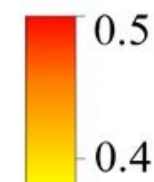

0.3

0.2

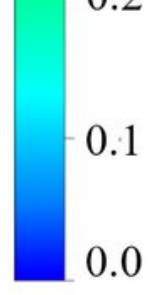

Figure 6

TAWSS and OSI characteristics for the FSI simulation. (a) $25 \%$ coarctation (b) $50 \%$ coarctation (c) $75 \%$ coarctation. 

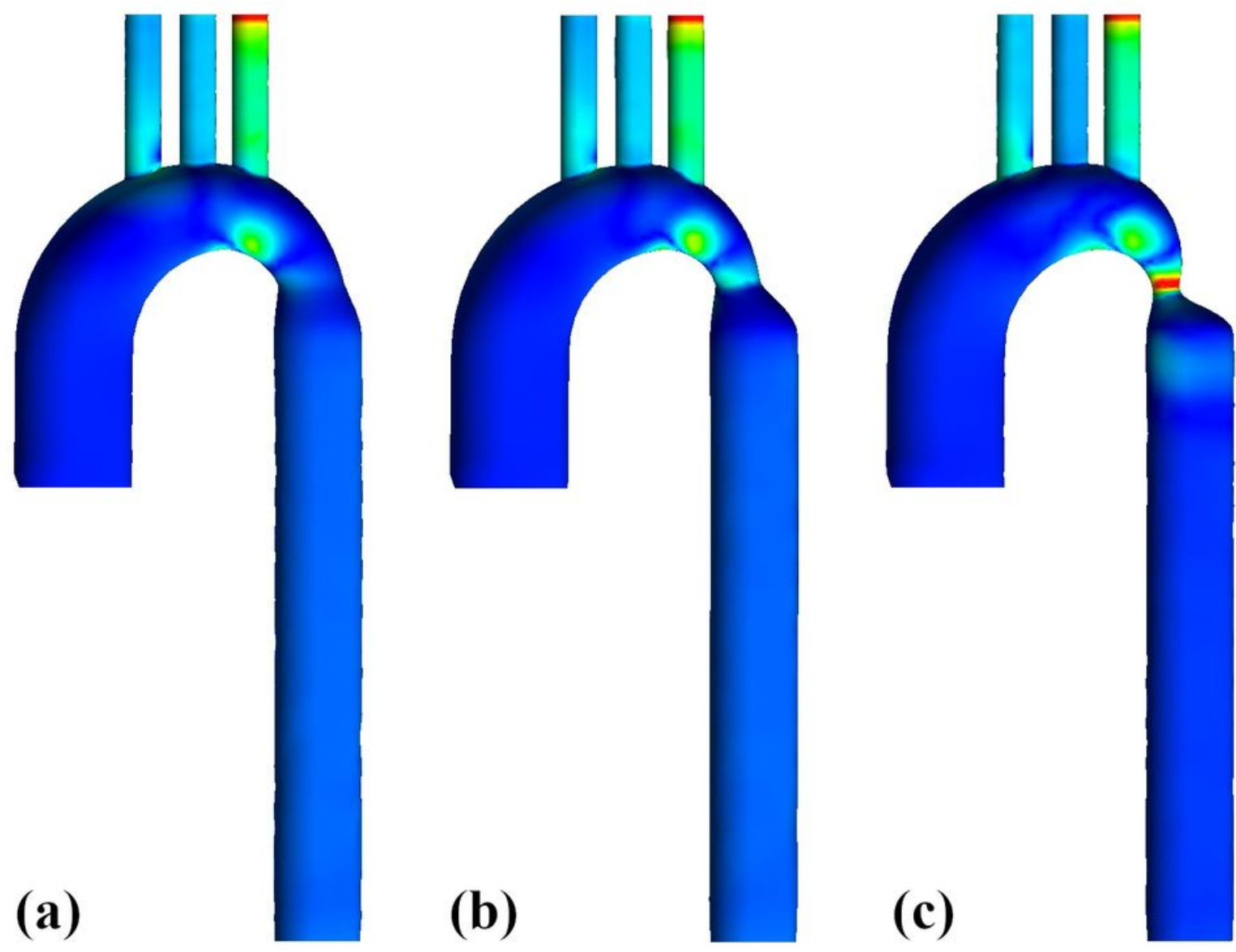

HOLMES

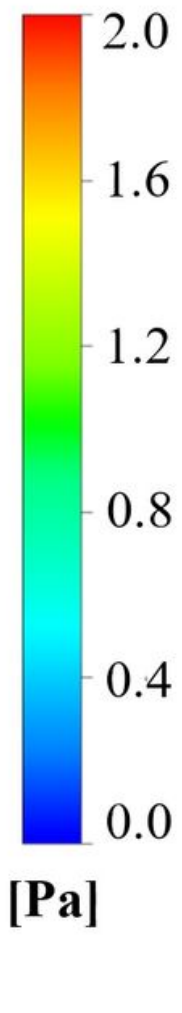

Figure 7

HOLMES characteristics. HOLMES distributions for the FSI simulation. (a) $25 \%$ coarctation (b) $50 \%$ coarctation (c) $75 \%$ coarctation. 
(a)
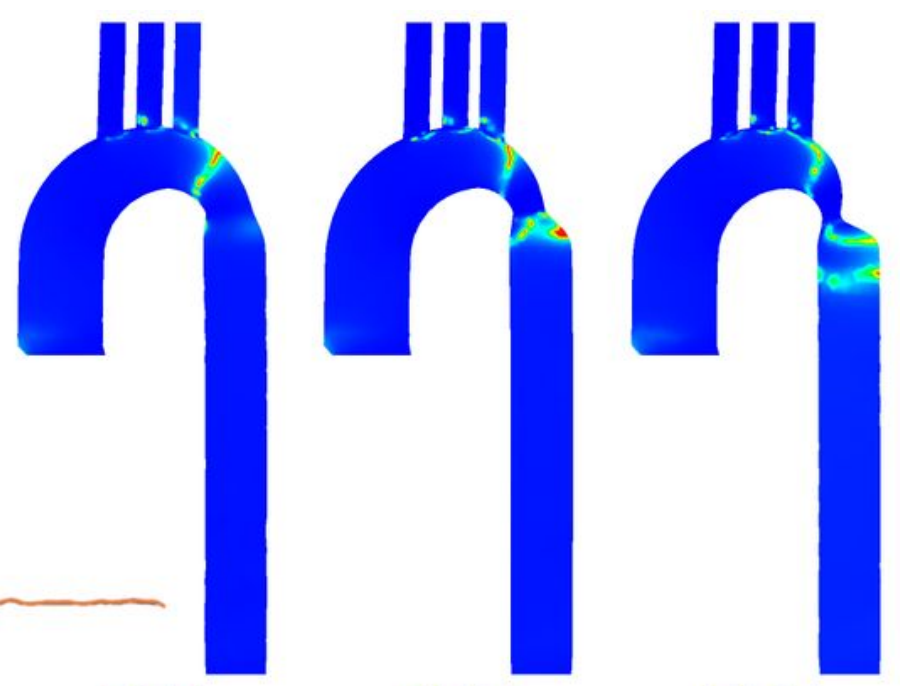

\section{RRT}

(b)
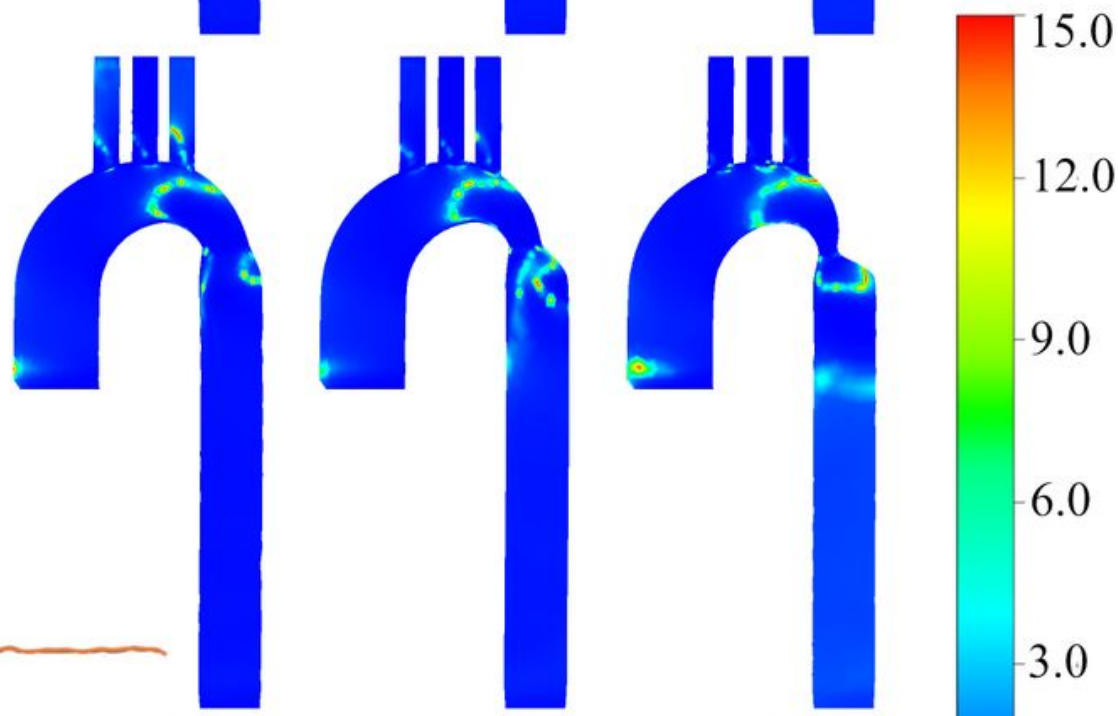

(c)
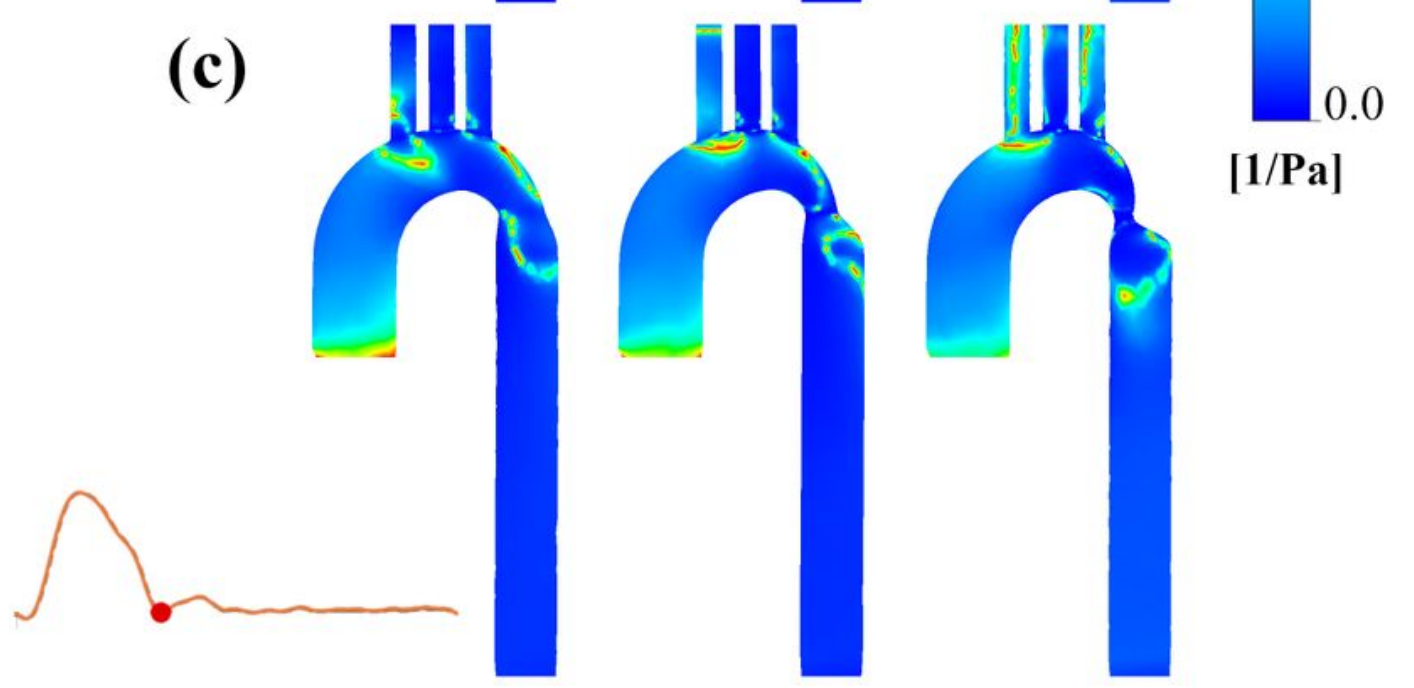

Figure 8

Contours of relative residence time (RRT) characteristics. RRT distributions for the FSI simulation (a) at mid-systole, (b) at peak systole and (c) at dicrotic notch (Left: $25 \%$ coarctation, middle: $50 \%$ coarctation, right: $75 \%$ coarctation) 